\title{
Geometric motivic Poincaré series of quasi-ordinary singularities
}

\author{
BY HELENA COBO PABLOS $\dagger$ \\ Department of Mathematics, University of Leuven, \\ Celestijnenlaan 200B, B-3001 Leuven-Heverlee, Belgium \\ AND PEDRO D. GONZÁLEZ PÉREZ ‡ \\ ICMAT. Depto. Álgebra. Universidad Complutense de Madrid. \\ Plaza de las Ciencias 3. 28040. Madrid. Spain
}

(Received )

\begin{abstract}
The geometric motivic Poincaré series of a germ $(S, 0)$ of complex algebraic variety takes into account the classes in the Grothendieck ring of the jets of arcs through $(S, 0)$. Denef and Loeser proved that this series has a rational form. We give an explicit description of this invariant when $(S, 0)$ is an irreducible germ of quasi-ordinary hypersurface singularity in terms of the Newton polyhedra of the logarithmic jacobian ideals. These ideals are determined by the characteristic monomials of a quasi-ordinary branch parametrizing $(S, 0)$.
\end{abstract}

\section{Introduction}

A germ $(S, 0)$ of complex analytic variety equidimensional of dimension $d$ is quasiordinary (q.o.) if there exists a finite map $\pi:(S, 0) \rightarrow\left(\mathbf{C}^{d}, 0\right)$ which is unramified outside a normal crossing divisor in $\left(\mathbf{C}^{d}, 0\right)$. Quasi-ordinary singularities admit fractional power series parametrizations, which generalize Newton-Puiseux expansions of plane curves (see [1). Quasi-ordinary surface singularities appear classically in the Jung's method to parametrize and resolve surface singularities (see [27]) and are related to the classification of singularities by Zariski's dimensionality type (see [30]). Classical examples are plane curve singularities, Hirzebruch-Jung surfaces and simplicial toric varieties. In addition to the applications in equisingularity problems, the class of q.o. singularities is also of interest to test and study various open questions and conjectures for singularities in general, particularly in the hypersurface case (see [32). In many cases the results passed by using the fractional power series parametrizations of these singularities, which allow explicit computations combining analytic, topological and combinatorial arguments (see for instance [29, 13, 37, 33, 2, 20, ). It is natural to investigate new invariants of singularities, such as those arising in the development of motivic integration, on this class of singularities with the hope to extend the methods or results to wider classes (for instance by passing through Jung's approach).

$\dagger$ Supported by Fundación Caja Madrid and MTM2004-08080-C02-01 grant of MEC Spain ‡ Supported by Programa Ramón y Cajal and MTM2004-08080-C02-01 grant of MEC Spain 
We recall the definition of the geometric motivic Poincaré series of a germ $(Z, 0)$ of complex algebraic variety (or complex analytic or algebroid) equidimensional of dimension $d$. The set $H_{Z}$ of formal arcs of the form, Spec $\mathbf{C}[[t]] \rightarrow(Z, 0)$ has a scheme structure over $\mathbf{C}$ (not necessarily of finite type). Let $s \geqslant 0$, the set $H_{s, Z}$ of $s$-jets of $(Z, 0)$ of the form Spec $\mathbf{C}[t] /\left(t^{s+1}\right) \rightarrow(Z, 0)$, has the structure of algebraic variety over $\mathbf{C}$. By a Theorem of Greenberg [22], the image of $H_{Z}$ by the natural morphism of schemes $j_{s}: H_{Z} \rightarrow H_{s, Z}$, which maps an arc to its $s$-jet, is a constructible subset of $H_{s, Z}$.

The Grothendieck ring $K_{0}\left(\operatorname{Var}_{\mathbf{C}}\right)$ of $\mathbf{C}$-varieties is generated by the symbols $[X]$ for $X$ an algebraic variety, subject to relations: $[X]=\left[X^{\prime}\right]$ if $X$ is isomorphic to $X^{\prime},[X]=$ $\left[X-X^{\prime}\right]+\left[X^{\prime}\right]$ if $X^{\prime}$ is closed in $X$ and $[X]\left[X^{\prime}\right]=\left[X \times X^{\prime}\right]$. Since a constructible set $W$ has an image $[W]$ in the Grothendieck ring $K_{0}\left(\operatorname{Var}_{\mathbf{C}}\right)$ of varieties, it is natural to consider the geometric motivic Poincaré series of the germ $(Z, 0)$ :

$$
P_{\text {geom }}^{(Z, 0)}(T):=\sum_{s \geqslant 0}\left[j_{s}\left(H_{Z}\right)\right] T^{s} \in K_{0}\left(\operatorname{Var}_{\mathbf{C}}\right)[[T]] .
$$

This series, which was introduced more generally by Denef and Loeser in [5], is inspired by related Poincaré series in arithmetic geometry [9]. It has a rational form:

Theorem. (see [5] Theorem 1.1) Set $\mathbf{L}:=\left[\mathbf{A}_{\mathbf{C}}^{1}\right]$. There exist $a_{i} \in \mathbf{Z}$ and $b_{i} \in \mathbf{Z}_{\geqslant 1}$, for $i=1, \ldots, r$ and $Q(T) \in K_{0}\left(\operatorname{Var}_{\mathbf{C}}\right)\left[\mathbf{L}^{-1}\right][T]$ such that

$$
P_{\text {geom }}^{(Z, 0)}(T)=Q(T) \prod_{i=1}^{r}\left(1-\mathbf{L}^{a_{i}} T^{b_{i}}\right)^{-1} \text { in } K_{0}\left(\operatorname{Var}_{\mathbf{C}}\right)\left[\mathbf{L}^{-1}\right][[T]] .
$$

There is not a general formula for this invariant in terms of a resolution of singularities of $(Z, 0)$ (notice that these kind of formulas exists for other motivic invariants as the motivic zeta function, see [6]). There is no conjecture on the meaning of the exponents $a_{i}, b_{i}$ which may appear in the denominator of a rational form of $P_{\text {geom }}^{(Z, 0)}(T)$. It is not known if such a rational expression for this series holds in the ring $K_{0}\left(\operatorname{Var}_{\mathbf{C}}\right)(T)$.

If $(Z, 0)$ is an analytically irreducible germ of plane curve the series $P_{\text {geom }}^{(Z, 0)}(T)$ determines and it is determined by the multiplicity of $(Z, 0)$ (see [7]). Nicaise has given formulas for $P_{\text {geom }}^{(Z, 0)}(T)$ for germs $(Z, 0)$ with a very special embedded resolution of singularities [35, for instance if $(Z, 0)$ is the cone over a smooth hypersurface $H \subset \mathbf{P}_{\mathbf{C}}^{d}$ then $P_{\text {geom }}^{(Z, 0)}(T)$ is determined by $[H], d$ and the multiplicity of $(Z, 0)$. Lejeune-Jalabert and Reguera have studied the motivic Poincare series of a germ $(Z, 0)$ of normal toric surface at its distinguished point. They have given a formula for the rational form of this series in terms of the Hirzebruch-Jung continued fraction describing the resolution of singularities of $Z$ (see [28] and also [34] for a different approach and comparison with other motivic series). If $(Z, 0)$ is a germ of affine toric variety of dimension $d$ we prove in 4 that $P_{\text {geom }}^{(Z, 0)}(T)$ is determined by the Newton polyhedra of the logarithmic jacobian ideals, which are defined in terms of the modules of differential forms with logarithmic poles outside the torus of $Z$. Rond studied the coefficients of the series $P_{\text {geom }}^{(S, 0)}(T)$ associated to a germ of q.o. hypersurface and computed the sum of this series in some particular cases (see [40]).

In this paper we describe the rational form of the geometric motivic Poincare series of a germ $(S, 0)$ of q.o. hypersurface singularity. Our approach is independent of Rond's.

A q.o. hypersurface singularity $(S, 0)$ has a fractional power series parametrization, which possesses a finite set of characteristic monomials (generalizing the characteristic 
exponents of plane branches) and which classify the embedded topological class of $(S, 0) \subset$ $\left(\mathbf{C}^{d+1}, 0\right)$ (see $\left.\mathbf{1 3}, \mathbf{2 9}\right)$. Since the normalization $(\bar{S}, 0)$ of the germ $(S, 0)$ is a toric singularity (see [16]) it is natural to extend the approach in [4] to the q.o. case. For this reason we introduce a sequence of monomial ideals $\mathcal{J}_{1}, \ldots, \mathcal{J}_{d}$ of the analytic algebra of the normalization $(\bar{S}, 0)$. These ideals, called the logarithmic jacobian ideals, are defined first in a combinatorial manner in terms of the characteristic monomials. Inspired by the toric case [4, 28, we prove that these ideals can be defined in terms of the composite of canonical maps $\Omega_{S} \rightarrow \Omega_{\bar{S}} \rightarrow \Omega_{\bar{S}}(\log D)$, where $\Omega_{S}\left(\right.$ resp. $\Omega_{\bar{S}}$ and $\left.\Omega_{\bar{S}}(\log D)\right)$ denotes the module of holomorphic differential forms of $(S, 0)$ (resp. over $(\bar{S}, 0)$ and the module of forms with logarithmic poles at the complement $D$ of the torus in $(\bar{S}, 0))$, see Section 9. In the toric case the blow up of the ideal $\mathcal{J}_{d}$ is the Nash modification (see [28]). In the q.o. case the normalized Nash modification of $(S, 0)$ is equal to the normalization of $(S, 0)$ followed by the normalized blow-up of the ideal $\mathcal{J}_{d}$ (see [17]).

We study the arc space $H_{S}$ of $(S, 0)$ by relating it with the arc space $H_{\bar{S}}$ of the normalization $(\bar{S}, 0)$. We denote by $H_{\bar{S}}^{*}$ (resp. by $H_{S}^{*}$ ) the set of arcs with generic point in the torus of $\bar{S}$ (resp. arcs in $H_{S}$ which lift to an $\operatorname{arc}$ in $H_{\bar{S}}^{*}$ ). For $s \geqslant 0$ we have that $j_{s}\left(H_{\bar{S}}\right)=j_{s}\left(H_{\bar{S}}^{*}\right)$ (see [34]) but $j_{s}\left(H_{S}\right) \neq j_{s}\left(H_{S}^{*}\right)$ in general if $S$ is not normal (the simplest example is Whitney umbrella). To avoid this difficulty we consider a finite set of q.o. coordinate sections $\left\{\left(S_{\theta}, 0\right)\right\}_{\theta}$ of $(S, 0)$, with $S_{\theta}=S$ if $\theta=0$, which are compatible with the toric structure of the normalization. We define an auxiliary motivic series $P(S)$ whose coefficients are the image in the Grothendieck ring of the $s$-jets of $\operatorname{arcs}$ in $H_{S}^{*}$ which are not jets of arcs through any proper q.o. coordinate section $\left(S_{\theta}, 0\right), \theta \neq 0$. It follows from this that $P_{\text {geom }}^{(S, 0)}(T)=\sum_{\theta} P\left(S_{\theta}\right)$. This reduces the study of $P_{\text {geom }}^{(S, 0)}(T)$ to the study of $P(S)$.

Since $(\bar{S}, 0)$ is a toric singularity, the arc space of the torus of $\bar{S}$ acts on $H_{\bar{S}}$. We use the orbit decomposition of $H_{\bar{S}}$ under this action studied by Ishii in [23, 24, 25]. The set $H_{S}^{*}$ decomposes as a disjoint union of orbits $H_{S, \nu}^{*}$, parametrized by the arc space of the torus, where $\nu$ runs through certain subset of the lattice $N$ of one-parametric subgroups of the torus. We prove that the $s$-jets of these orbits are locally closed subsets which are either disjoint or equal and we characterize the equality in combinatorial terms. Then we prove that the coefficient of $T^{m}$ in the series $P(S)$ expand as a sum of classes $\left[j_{m}\left(H_{S, \nu}^{*}\right)\right]$, where $\nu$ runs through a finite subset of the lattice $N$.

The description of the rational form of the geometric motivic Poincaré series $P_{\text {geom }}^{(S, 0)}(T)$, is done by using the methods and combinatorial results of [4].

We determine first a formula for the class $\left[j_{m}\left(H_{S, \nu}^{*}\right)\right]$ in terms of the Newton polyhedra of the logarithmic jacobian ideals in Theorem [5.8. These ideals satisfy similar combinatorial properties in the toric case and in the q.o. case, though the proofs are more difficult in the second case. In addition, new combinatorial features are needed to relate the parametrization of $(S, 0)$ with fractional power series with the parametrization of $j_{m}\left(H_{S, \nu}^{*}\right)$ by using the coordinates of the arc space of the torus.

We prove then that the rational form of the series $P(S)$ is determined by the Newton polyhedra of the logarithmic jacobian ideals and the lattice $N$ (see Theorem [5.9). We deduce a formula for the rational form of $P_{\text {geom }}^{(S, 0)}(T)$, which holds in the ring $\mathbf{Z}[\mathbf{L}](T) \subset$ $K_{0}\left(\operatorname{Var}_{\mathbf{C}}\right)(T)$, see Corollary 5.10. In particular a finite set of candidate poles of $P_{\text {geom }}^{(S, 0)}(T)$ is obtained. Our result implies that the embedded topological type of the germ $(S, 0) \subset$ $\left(\mathbf{C}^{d+1}, 0\right)$ determines the series $P_{\text {geom }}^{(S, 0)}(T)$. The converse is not true even if $d=1$. As an 
application we deduce a formula for the motivic volume of the arc space of a q.o. hypersurface germ $(S, 0)$ in terms of the logarithmic jacobian ideal $\mathcal{J}_{d}$ (see Corollary [5.11).

Notice that the series $P_{\text {geom }}^{(S, 0)}(T)$ and $P_{\text {geom }}^{(\bar{S}, 0)}(T)$ may be quite different (for instance if the normalization of $(S, 0)$ is smooth). In Section 10 we give an example of q.o. surface $(S, 0)$ such that $P_{\text {geom }}^{(S, 0)}(T) \neq P_{\text {geom }}^{(Z(S), 0)}(T)$, where $Z(S)$ is the monomial variety associated to the q.o. hypersurface (see Section 3 ). Notice that these two series coincide in the one dimensional case.

The paper is organized as follows. The first three sections introduce the basic notions we need on arc and jets spaces, toric geometry and q.o. singularities. We describe the orbit decomposition of the arc space of a q.o. hypersurface in Section 4 . In Section 5 we state the main results. In Section [6] we give some combinatorial convexity properties of the Newton polyhedra of the logarithmic jacobian ideals. In Section 7 we prove Theorem 5.8 In Section 8 we describe the series $P(S)$ and prove the rationality results. In Section 9 we define the logarithmic jacobian ideals in terms of differential forms.

The results and proofs in this paper hold if the field $\mathbf{C}$ is replaced by any algebraically closed field of characteristic zero.

\section{Basic definitions on arc and jet spaces}

We refer to [26, 10, 6, 31, 41 for expository papers on arc and jet schemes and/or motivic integration. We introduce arc and jet spaces on an equidimensional germ of complex analytic variety $(Z, 0)$ (or complex algebraic variety or algebroid). Arc and jet spaces can be defined on any algebraic variety (without fixing the origin of the arcs).

We have an embedding $(Z, 0) \subset\left(\mathbf{A}_{\mathbf{C}}^{n}, 0\right)$ in such a way that the germ $(Z, 0)$ is defined by the ideal $I \subset \mathbf{C}\left\{x_{1}, \ldots, x_{n}\right\}$. An $\operatorname{arc} h: \operatorname{Spec} \mathbf{C}[[t]] \rightarrow\left(\mathbf{A}_{\mathbf{C}}^{n}, 0\right)$ (resp. a $m$-jet Spec $\left.\mathbf{C}[t] /\left(t^{m+1}\right) \rightarrow(Z, 0)\right)$ is defined by $n$ formal power series

$$
x_{i}(t)=a_{i}^{(1)} t+a_{i}^{(2)} t^{2}+\cdots+a_{i}^{(r)} t^{r}+\cdots, \quad i=1, \ldots, n,
$$

(resp. $n$-polynomial expressions (1.1) $\bmod t^{m+1}$ ). For any $F \in I$, the coefficient of $t^{k}$ in the series $F\left(x_{1}(t), \ldots, x_{n}(t)\right)$ is a polynomial expression $\alpha_{F}^{(k)}\left(\underline{a}^{(1)}, \ldots, \underline{a}^{(k)}\right)$, where $\underline{a}^{(j)}:=\left(a_{1}^{(j)}, \ldots, a_{n}^{(j)}\right)$, for $j \in \mathbf{Z}_{\geqslant 0}$. This arc (resp. $m$-jet) factors through $(Z, 0)$ if for any $F \in I$ we have $F\left(x_{1}(t), \ldots, x_{n}(t)\right)=0$. (resp. $\left.F\left(x_{1}(t), \ldots, x_{n}(t)\right)=0 \bmod t^{m+1}\right)$.

The arc space $H_{Z}$ (resp. $m$-jet space $H_{m, Z}$ ) is the reduced scheme underlying the affine scheme Spec $\mathcal{A}_{Z}$, where $\mathcal{A}_{Z}=\mathbf{C}\left[\underline{a}^{(1)}, \underline{a}^{(2)}, \underline{a}^{(3)}, \ldots\right] /\left(\alpha_{F}^{(k)}\left(\underline{a}^{(1)}, \ldots, \underline{a}^{(k)}\right)\right)_{k \geqslant 1, F \in I}$ (resp. Spec $\mathcal{A}_{m, Z}$, where $\left.\mathcal{A}_{m, Z}:=\mathbf{C}\left[\underline{a}^{(1)}, \ldots, \underline{a}^{(m)}\right] /\left(\alpha_{F}^{(k)}\left(\underline{a}^{(1)}, \ldots, \underline{a}^{(k)}\right)\right)_{k=1, \ldots, m, F \in I}\right)$.

We have morphisms of schemes $j_{m}: H_{Z} \rightarrow H_{m, Z}$ and $j_{m}^{m+1}: H_{m+1, Z} \rightarrow H_{m, Z}$ induced by truncating arcs or jets $\bmod t^{m+1}$, for every $m \geqslant 0$. We have that $H_{Z}=\varliminf_{m} H_{m, Z}$.

If $h(t)=\sum_{i \geqslant 0} a_{i} t^{i}$ is a formal power series and $m \geqslant 0$ we set $j_{m}(h):=h(t) \overleftrightarrow{\bmod t^{m+1}}$.

\section{Some basic definitions on toric geometry}

See [12, 11, 36 for general references on toric geometry. If $N \cong \mathbf{Z}^{d}$ is a lattice we denote by $N_{\mathbf{R}}$ (resp. $N_{\mathbf{Q}}$ ) the vector space spanned by $N$ over the field $\mathbf{R}$ (resp. over $\mathbf{Q}$ ). If $\left\{u_{i}\right\}_{i \in I} \subset N_{\mathbf{R}}$ we denote by $\operatorname{span}_{\mathbf{Q}}\left\{u_{i}\right\}_{i \in I}$ the linear subspace spanned by the $u_{i}$ over Q.

In what follows a cone mean a rational convex polyhedral cone: the set of non negative linear combinations of vectors $a_{1}, \ldots, a_{r} \in N$. The cone $\tau$ is strictly convex if it contains no lines, in that case we denote by 0 the 0 -dimensional face of $\tau$. The dual cone $\tau^{\vee}$ (resp. 
orthogonal cone $\tau^{\perp}$ ) of $\tau$ is the set $\left\{w \in M_{\mathbf{R}} \mid\langle w, u\rangle \geqslant 0\right.$, (resp. $\left.\left.\langle w, u\rangle=0\right) \forall u \in \tau\right\}$. We denote by $\stackrel{\circ}{\tau}$ or by $\operatorname{int}(\tau)$ the relative interior of the cone $\tau$. A fan $\Sigma$ is a family of strictly convex cones in $N_{\mathbf{R}}$ such that any face of such a cone is in the family and the intersection of any two of them is a face of each. The relation $\theta \leqslant \tau$ (resp. $\theta<\tau$ ) denotes that $\theta$ is a face of $\tau$ (resp. $\theta \neq \tau$ is a face of $\tau$ ). The support (resp. the $k$-skeleton) of the fan $\Sigma$ is the set $|\Sigma|:=\bigcup_{\tau \in \Sigma} \tau \subset N_{\mathbf{R}}$ (resp. $\Sigma^{(k)}=\{\tau \in \Sigma \mid \operatorname{dim} \tau=k\}$ ). We say that a fan $\Sigma^{\prime}$ is a subdivision of the fan $\Sigma$ if both fans have the same support and if every cone of $\Sigma^{\prime}$ is contained in a cone of $\Sigma$. If $\Sigma_{i}$ for $i=1, \ldots, n$ are fans with the same support their intersection $\cap_{i=1}^{n} \Sigma_{i}:=\left\{\cap_{i=1}^{n} \tau_{i} \mid \tau_{i} \in \Sigma_{i}\right\}$ is also a fan.

Let $\tau$ be a strictly convex cone rational for the lattice $N$. The toric variety $Z_{\tau}:=$ Spec $\mathbf{C}\left[\tau^{\vee} \cap M\right]$, denoted also by $Z_{\tau, N}$ or $Z^{\tau^{\vee} \cap M}$, is normal. The torus $T_{N}:=Z^{M}$ is an open dense subset of $Z_{\tau}$, which acts on $Z_{\tau}$ and the action extends the action of the torus on itself by multiplication. There is a one to one correspondence between the faces $\theta$ of $\tau$ and the orbits $\operatorname{orb}_{\theta}$ of the torus action on $Z_{\tau}$, which reverses the inclusions of their closures. The closure of $\operatorname{orb}_{\theta}$ is the toric variety $Z^{\sigma^{\vee} \cap \theta^{\perp} \cap M}$ for $\tau \leqslant \sigma$. The orbit orb $\operatorname{or}_{\tau}$ is reduced to a closed point called the origin 0 of the toric variety $Z_{\tau}$. The $\operatorname{ring} \mathbf{C}\left\{\tau^{\vee} \cap M\right\}$ of germs of holomorphic functions at $0 \in Z_{\tau}$ is a subring of the ring $\mathbf{C}\left[\left[\tau^{\vee} \cap M\right]\right]$ of formal power series with exponents in $\tau^{\vee} \cap M$.

If $\emptyset \neq \mathcal{I} \subset \tau^{\vee} \cap M$, the Newton polyhedron $\mathcal{N}(\mathcal{I})$ of the monomial ideal defined by $\mathcal{I}$ is the Minkowski sum of sets $\mathcal{I}+\tau^{\vee}$. The support function $\operatorname{ord}_{\mathcal{I}}$ of the polyhedron $\mathcal{N}(\mathcal{I})$ is defined by $\operatorname{ord}_{\mathcal{I}}: \tau \rightarrow \mathbf{R}, \nu \mapsto \inf _{\omega \in \mathcal{N}(\mathcal{I})}\langle\nu, \omega\rangle$. A vector $\nu \in \tau$ defines the face $\mathcal{F}_{\nu}:=\left\{\omega \in \mathcal{N}(\mathcal{I}) \mid\langle\nu, \omega\rangle=\operatorname{ord}_{\mathcal{I}}(\nu)\right\}$ of the polyhedron $\mathcal{N}(\mathcal{I})$. All faces of $\mathcal{N}(\mathcal{I})$ are of this form, the compact faces are defined by vectors $\nu \in \stackrel{\circ}{\tau}$. The dual fan $\Sigma(\mathcal{I})$ associated to an integral polyhedron $\mathcal{N}(\mathcal{I})$ is a fan supported on $\tau$ which is formed by the cones $\sigma(\mathcal{F}):=\left\{\nu \in \sigma \mid\langle\nu, \omega\rangle=\operatorname{ord}_{\mathcal{I}}(\nu), \forall \omega \in \mathcal{F}\right\}$, for $\mathcal{F}$ running through the faces of $\mathcal{N}(\mathcal{I})$. Notice that if $\theta \in \Sigma(\mathcal{I})$ and if $\nu, \nu^{\prime} \in \stackrel{\circ}{\theta}$ then we have that $\mathcal{F}_{\nu}=\mathcal{F}_{\nu^{\prime}}$ and we denote this face of $\mathcal{N}(\mathcal{I})$ also by $\mathcal{F}_{\theta}$.

The affine varieties $Z_{\sigma}$ corresponding to cones in a fan $\Sigma$ glue up to define a toric variety $Z_{\Sigma}$. The subdivision $\Sigma^{\prime}$ of a fan $\Sigma$ defines a toric modification $\pi_{\Sigma^{\prime}}: Z_{\Sigma^{\prime}} \rightarrow Z_{\Sigma}$.

If $\mathcal{I}$ is a monomial ideal of $Z_{\tau} \Sigma=\Sigma(\mathcal{I})$, the toric modification $\pi_{\Sigma}: Z_{\Sigma} \rightarrow Z_{\tau}$ is the normalized blowing up of $Z_{\tau}$ centered at $\mathcal{I}$ (see [28 for instance).

\section{Quasi-ordinary hypersurface singularities}

A germ $(S, 0)$ of complex analytic variety equidimensional of dimension $d$ is quasiordinary (q.o.) if there exists a finite projection $\pi:(S, 0) \rightarrow\left(\mathbf{C}^{d}, 0\right)$ which is a local isomorphism outside a normal crossing divisor. If $(S, 0)$ is a hypersurface there is an embedding $(S, 0) \subset\left(\mathbf{C}^{d+1}, 0\right)$, defined by an equation $f=0$, where $f \in \mathbf{C}\left\{x_{1}, \ldots, x_{d}\right\}\left[x_{d+1}\right]$ is a q.o. polynomial: a Weierstrass polynomial with discriminant $\Delta_{x_{d+1}} f$ of the form $\Delta_{x_{d+1}} f=x^{\delta} \epsilon$ for a unit $\epsilon$ in the ring $\mathbf{C}\{x\}$ of convergent power series in the variables $x=\left(x_{1}, \ldots, x_{d}\right)$ and $\delta \in \mathbf{Z}_{\geqslant 0}^{d}$.

We suppose that $(S, 0)$ is analytically irreducible, that is $f \in \mathbf{C}\left\{x_{1}, \ldots, x_{d}\right\}\left[x_{d+1}\right]$ is irreducible. The Jung-Abhyankar theorem guarantees that the roots of a q.o. polynomial $f$, called q.o. branches, are fractional power series in $\mathbf{C}\left\{x^{1 / n_{0}}\right\}$, for $n_{0}=\operatorname{deg} f$ (see [1]).

Lemma 3·1. (see [13, Prop. 1.3). Let $f \in \mathbf{C}\left\{x_{1}, \ldots, x_{n}\right\}\left[x_{d+1}\right]$ be an irreducible 
q.o. polynomial. Let $\zeta$ be a root of $f$ with expansion:

$$
\zeta=\sum \beta_{\lambda} x^{\lambda}
$$

There exists $0 \neq \lambda_{1}, \ldots, \lambda_{g} \in \mathbf{Q}_{\geqslant 0}^{d}$ such that if $M_{0}:=\mathbf{Z}^{d}$ and $M_{j}:=M_{j-1}+\mathbf{Z} \lambda_{j}$ for $j=1, \ldots, g$, then:

(i) $\beta_{\lambda_{i}} \neq 0$ and if $\beta_{\lambda} \neq 0$ then $\lambda \in M_{j}$ where $j$ is the unique integer such that $\lambda_{j} \leqslant \lambda$ and $\lambda_{j+1} \not \lambda$ (where $\leqslant$ means coordinate-wise and we convey that $\lambda_{g+1}=\infty$ ).

(ii) For $j=1, \ldots, g$, we have $\lambda_{j} \notin M_{j-1}$, hence the index $n_{j}=\left[M_{j-1}: M_{j}\right]$ is $>1$.

Definition 3.2. The exponents $\lambda_{1}, \ldots, \lambda_{g}$ in Lemma 3.1 above (resp. the monomials $\left.x^{\lambda_{1}}, \ldots, x^{\lambda_{g}}\right)$ are called characteristic of the q.o. branch $\zeta$. We denote by $M$ the lattice $M_{g}$ and we call it the lattice associated to the q.o. branch $\zeta$. We denote its dual lattice by $N$. For convenience we denote $\lambda_{0}:=0$.

Without loss of generality we relabel the variables $x_{1}, \ldots, x_{d}$ in such a way that if $\lambda_{j}=\left(\lambda_{j}^{1}, \ldots, \lambda_{j}^{d}\right) \in \mathbf{Q}^{d}$ for $j=1, \ldots, g$, then we have:

$$
\left(\lambda_{1}^{1}, \ldots, \lambda_{g}^{1}\right) \geqslant_{\text {lex }} \cdots \geqslant_{\text {lex }}\left(\lambda_{1}^{d}, \ldots, \lambda_{g}^{d}\right),
$$

where $\geqslant_{\text {lex }}$ is lexicographic order. The q.o. branch $\zeta$ is normalized if $\lambda_{1}$ is not of the form $\left(\lambda_{1}^{1}, 0, \ldots, 0\right)$ with $\lambda_{1}^{1}<1$. Lipman proved that the germ $(S, 0)$ can be parametrized by a normalized q.o. branch (see [13, Appendix). We assume from now on that the q.o. branch $\zeta$ is normalized.

The semigroup $\mathbf{Z}_{\geqslant 0}^{d}$ has a minimal set of generators $e_{1}, \ldots, e_{d}$ which is a basis of the lattice $M_{0}$. The dual basis of the dual lattice $N_{0}$ spans a regular cone $\sigma$ in $N_{0, \mathbf{R}}$. It follows that $\mathbf{Z}_{\geqslant 0}^{d}=\sigma^{\vee} \cap M_{0}$, where $\sigma^{\vee}=\mathbf{R}_{\geqslant 0}^{d}$ is the dual cone of $\sigma$. The $\mathbf{C}$-algebra $\mathbf{C}\left\{x_{1}, \ldots, x_{d}\right\}$ is isomorphic to $\mathbf{C}\left\{\sigma^{\vee} \cap M_{0}\right\}$. This isomorphism sends the monomial $x_{1}^{\alpha_{1}} \cdots x_{d}^{\alpha_{d}}$ in the monomial $X^{\alpha} \in \mathbf{C}\left\{\sigma^{\vee} \cap M_{0}\right\}$ if $\alpha=\sum_{i=1}^{d} \alpha_{i} e_{i}$. The local algebra $\mathcal{O}_{S}=$ $\mathbf{C}\left\{x_{1}, \ldots, x_{d}\right\}\left[x_{d+1}\right] /(f)$ of the singularity $(S, 0)$ is isomorphic to $\mathbf{C}\left\{\sigma^{\vee} \cap M_{0}\right\}[\zeta]$. By Lemma $3 \cdot 1$ the series $\zeta$ can be viewed as an element $\sum \beta_{\lambda} X^{\lambda}$ of the algebra $\mathbf{C}\left\{\sigma^{\vee} \cap M\right\}$.

LEMma 3·3. (See [16]). The homomorphism $\mathcal{O}_{S} \rightarrow \mathbf{C}\left\{\sigma^{\vee} \cap M\right\}$ is the inclusion of $\mathcal{O}_{S}$ in its integral closure in its field of fractions.

The previous Lemma shows that the normalization $(\bar{S}, 0)$ of a q.o. hypersurface singularity $(S, 0)$ is the germ of the toric variety $\bar{S}=Z^{\sigma^{\vee} \cap M}$ at the distinguished point. More generally, the normalization of a q.o. singularity, non necessarily hypersurface, is a toric singularity (see [37] and [38). If $n:(\bar{S}, 0) \rightarrow(S, 0)$ is the normalization map the composite

$$
(\bar{S}, 0) \stackrel{n}{\rightarrow}(S, 0) \stackrel{\pi}{\rightarrow}\left(\mathbf{C}^{d}, 0\right),
$$

is a q.o. projection, since it is the toric map defined by the inclusion of algebras, $\mathbf{C}\left\{\sigma^{\vee} \cap\right.$ $\left.M_{0}\right\} \subset \mathbf{C}\left\{\sigma^{\vee} \cap M\right\}$, induced by the finite index lattice extension $M_{0} \subset M$ (see [36 ).

If $\theta \leqslant \sigma$, the toric map between the orbit closures $Z^{\sigma^{\vee} \cap M \cap \theta^{\perp}} \rightarrow Z^{\sigma^{\vee} \cap M_{0} \cap \theta^{\perp}}$ is the composite of

$$
\left(Z^{\sigma^{\vee} \cap M \cap \theta^{\perp}}, 0\right) \stackrel{n_{\theta}}{\rightarrow}\left(S_{\theta}, 0\right) \stackrel{\pi_{\theta}}{\rightarrow}\left(Z^{\sigma^{\vee} \cap M_{0} \cap \theta^{\perp}}, 0\right),
$$

where $n_{\theta}$ and $\pi_{\theta}$ denote respectively the restrictions of $n$ and $\pi$ and $\left(S_{\theta}, 0\right)$ is the coordinate section of $(S, 0)$ given by: $S_{\theta}:=S \cap\left\{x_{j}=0 \mid\right.$ for $1 \leqslant j \leqslant d$ and $\left.e_{j} \notin \theta^{\perp}\right\}$. It follows that $\left(S_{\theta}, 0\right)$ is q.o. (see [29]). The germ $\left(S_{\theta}, 0\right)$ is a q.o. hypersurface of dimension $\operatorname{dim} \theta^{\perp}$ parametrized by the series $\zeta_{\theta}:=\sum_{\lambda \in \sigma^{\vee} \cap \theta^{\perp}} \beta_{\lambda} X^{\lambda}$. 
Definition 3.4. For $\theta \leqslant \sigma$, we call $S_{\theta}$ the q.o. coordinate section associated to $\theta$, we denote by $M(\theta, \zeta)$ the lattice associated to the q.o. branch $\zeta_{\theta}$ and by $N(\theta, \zeta)$ its dual lattice.

Notice that the dual cone of $\sigma^{\vee} \cap \theta^{\perp}$ is equal to the image $\sigma / \theta \mathbf{R}$ of the cone $\sigma$ in the quotient vector space $N_{\mathbf{R}} / \theta \mathbf{R}$. We have finite index lattice extensions $M_{0}(\theta):=$ $M_{0} \cap \theta^{\perp} \hookrightarrow M(\theta, \zeta) \hookrightarrow M(\theta):=M \cap \theta^{\perp}$. The map $n_{\theta}$ in (3.4) is a ramified covering with $\left[M \cap \theta^{\perp}: M(\theta, \zeta)\right]$ sheets, which is unramified over the torus. The index $\left[M \cap \theta^{\perp}: M(\theta, \zeta)\right]$ is equal to the number of irreducible components of the germ of $S$ at a generic point of $S_{\theta}$ (cf. Proposition 4.5.4 in [29).

The elements of $M$ defined by: $\gamma_{1}=\lambda_{1}$ and $\gamma_{j+1}-n_{j} \gamma_{j}=\lambda_{j+1}-\lambda_{j}$ for $j=1, \ldots, g-1$, span the semigroup $\Gamma:=\mathbf{Z}_{\geqslant 0}^{d}+\gamma_{1} \mathbf{Z}_{\geqslant 0}+\cdots+\gamma_{g} \mathbf{Z}_{\geqslant 0} \subset \sigma^{\vee} \cap M$. The semigroup $\Gamma$ defines an analytic invariant of the germ $(S, 0)$ (see [15, 18, 37]).

Definition $3 \cdot 5$. The monomial variety associated to $(S, 0)$ is the toric variety $Z(S):=$ Spec $\mathbf{C}[\Gamma]$.

Following Teissier's approach [14, [39], the singularity $(S, 0)$ can be presented, after re-embedding in a suitable affine space of larger dimension, as the generic fiber of a 1-parametrical deformation with special fiber equal to the monomial variety $Z(S)$ (see [16]). This family is equisingular in the sense that one toric morphism of the affine space provides a simultaneous embedded resolution of singularities of the family (see [14 for the one dimensional case, [16] in the q.o. case and [39] for related results in a more general context). The normalizations of $(S, 0)$ and of $(Z(S), 0)$ coincide. See [16, 17] for the properties of the equisingular deformation of $Z(S)$ with generic fiber $(S, 0)$.

\section{Arcs and jets on a quasi-ordinary hypersurface}

In this Section we study the arcs in the q.o. hypersurface $(S, 0)$ by using the toric structure of $(\bar{S}, 0)$, following the approach of $[\mathbf{2 5}, \mathbf{2 4}, 4$. We keep notations of Section 3. Recall that the normalization $\bar{S}$ of $S$ is equal to the toric variety $Z^{\sigma^{\vee} \cap M}$. The set $H_{\bar{S}}^{*}$ of arcs of $H_{\bar{S}}$ with generic point in the torus is

$$
H_{\bar{S}}^{*}=\left\{h \in H_{\bar{S}} \mid X^{e} \circ h \neq 0, \forall e \in \sigma^{\vee} \cap M\right\} .
$$

Any $\operatorname{arc} \bar{h} \in H_{\bar{S}}^{*}$ defines two group homomorphisms $\nu_{\bar{h}}: M \rightarrow \mathbf{Z}$ and $\omega_{\bar{h}}: M \rightarrow \mathbf{C}[[t]]^{*}$ by

$$
X^{m} \circ \bar{h}=t^{\nu_{\bar{h}}(m)} \omega_{\bar{h}}(m) \text {, for } m \in M .
$$

If $m \in \sigma^{\vee} \cap M$ then we have that $\nu_{\bar{h}}(m)>0$ hence $\nu_{\bar{h}}$ belongs to $\stackrel{\circ}{\sigma} \cap N$. It follows that $\omega_{\bar{h}}$ defines an arc in the torus $T_{N}$ (see [4]). We define similarly the set $H_{S}^{*} \subset H_{S}$ :

Definition 4·1. (see [25, 24, 4]) $H_{S}^{*}=\left\{h \in H_{S} \mid x_{i} \circ h \neq 0, i=1, \ldots, d+1\right\}$.

The set $H_{S}^{*}$ consists of those arcs $h \in H_{S}$ such that there exists $\bar{h} \in H_{\bar{S}}^{*}$ such that $h=n \circ \bar{h}$. Notice that if $h \in H_{S}^{*}$ then the generic point of $h$ is not contained in the singular locus Sing $S$ of $S$, since Sing $S \cap \pi^{-1}\left(\left(\mathbf{C}^{*}\right)^{d}\right)=\emptyset$ by definition of $\pi$. By the valuative criterion of properness applied to the normalization map, there exists a unique $\operatorname{arc} \bar{h} \in H_{\bar{S}}$ such that $h=n \circ \bar{h}$. Since (3.3) defines a q.o. projection it follows that $\bar{h} \in H_{\bar{S}}^{*}$ and $h \mapsto \bar{h}$ defines a bijective correspondence between the sets $H_{S}^{*}$ and $H_{\bar{S}}^{*}$. 
Definition $4 \cdot 2$. For any $\nu \in \stackrel{\circ}{\sigma} \cap N$ we define the sets:

$$
H_{\bar{S}, \nu}^{*}=\left\{\bar{h} \in H_{\bar{S}}^{*} \mid \nu_{\bar{h}}=\nu\right\} \text { and } H_{S, \nu}^{*}=\left\{h \in H_{S}^{*} \mid \exists \bar{h} \in H_{\bar{S}}^{*}: n \circ \bar{h}=h, \nu_{\bar{h}}=\nu\right\} .
$$

Ishii noticed that the space of arcs in the torus acts on the arc space of a toric variety (see [23, 24]). The set $H_{\bar{S}, \nu}^{*}$ is an orbit of the action of the arc space of the torus of $\bar{S}$ (see [23]). The map $\bar{h} \mapsto n \circ \bar{h}$ defines a bijective correspondence between the sets $H_{\bar{S}, \nu}^{*}$ and $H_{S, \nu}^{*}$. We usually denote the set $H_{S, \nu}^{*}$ by $H_{\nu}^{*}$. The set $H_{\bar{S}}^{*}$ is invariant for this action of the arc space of the torus and we obtain the partitions: $H_{\bar{S}}^{*}=\bigsqcup_{\nu \in \sigma \cap N} H_{\bar{S}, \nu}^{*}$ and $H_{S}^{*}=\bigsqcup_{\nu \in \circ \cap N} H_{S, \nu}^{*}$.

Notice that $H_{S}^{*}$ can be defined also as the set of arcs $h \in H_{S}$ such that the arc $\pi \circ h \in H_{\mathbf{A}_{\mathbf{C}}^{d}}$ has generic point in the torus, where we consider the affine space $\mathbf{A}_{\mathbf{C}}^{d}$ with the structure of toric variety $\mathbf{A}_{\mathbf{C}}^{d}=Z^{\sigma^{\vee} \cap M_{0}}$. If $h \in H_{S}$ then $\pi \circ h$ factors through a unique minimal orbit closure, of the form $Z^{\sigma^{\vee} \cap \theta^{\perp} \cap M_{0}}$, in such a way that $\pi \circ h$ has generic point in the torus of $Z^{\sigma^{\vee} \cap \theta^{\perp} \cap M_{0}}$. In this case, it follows from the properties of (3.3) that the arc $h$ factors through $\left(S_{\theta}, 0\right)$ and belongs to the set $H_{S_{\theta}}^{*}$. We deduce from this observations the following partition of the arc space $H_{S}=\bigsqcup_{\theta \leqslant \sigma} H_{S_{\theta}}^{*}$.

\section{Statement of the main results}

We state in this section the main results of the paper. We keep notations of the previous sections.

Notation $5 \cdot 1$. We denote by $e_{1}, \ldots, e_{d}$ the elements of the canonical basis of $\mathbf{Z}^{d}$. We also denote the characteristic exponent $\lambda_{j}$ of the q.o. branch $\zeta$ by $e_{d+j}$, for $j=1, \ldots, g$. We set $e_{0}:=\infty$ and $e_{d+g+1}:=\infty$.

Definition $5 \cdot 2$. We introduce the following subsets of $\sigma^{\vee} \cap M$ associated to the q.o. branch $\zeta$ parametrizing the germ $(S, 0)$.

$$
\mathcal{J}_{k}:=\left\{e_{j_{1}}+\cdots+e_{j_{k}} \mid e_{j_{1}} \wedge \cdots \wedge e_{j_{k}} \neq 0,1 \leqslant j_{1}, \ldots, j_{k-1} \leqslant d, \text { and } 1 \leqslant j_{k} \leqslant d+g\right\} .
$$

We call the monomial ideal of $\mathbf{C}\left\{\sigma^{\vee} \cap M\right\}$ defined by (5.1) the $k^{\text {th }}$-logarithmic jacobian ideal of $(S, 0)$ relative to the q.o. projection $\pi$, for $k=1, \ldots, d$. We abuse slightly of notation by denoting this ideal also by $\mathcal{J}_{k}$. We denote by $\Sigma_{k}$ (resp. by ord $\mathcal{J}_{k}$ ) the dual subdivision of $\sigma$ (resp. the support function) associated to the Newton polyhedron of the $k^{t h}$-logarithmic jacobian ideal $\mathcal{J}_{k}$, for $k=1, \ldots, d$.

Remark $5 \cdot 3$. These ideals can be defined in terms of holomorphic differential forms with logarithmic poles outside the torus of $\bar{S}$ (see Section 9). The terminology is inspired by 28 and 4 .

Now we introduce an auxiliary series to study $P_{\text {geom }}^{(S, 0)}(T)$. By Greenberg's Theorem, for any $\theta \leqslant \sigma$ we have that $j_{s}\left(H_{S_{\theta}}\right)$ is a constructible subset of the $s$-jet space of $(S, 0)$. It follows that

$$
j_{s}\left(H_{S}\right) \backslash \bigcup_{0 \neq \theta \leqslant \sigma} j_{s}\left(H_{S_{\theta}}^{*}\right)=j_{s}\left(H_{S}\right) \backslash \bigcup_{0 \neq \theta \leqslant \sigma} j_{s}\left(H_{S_{\theta}}\right)
$$

is also a constructible subset. Taking the images of theses sets in the Grothendieck ring it is natural to consider the auxiliary Poincaré series,

$$
P(S):=\sum_{s \geqslant 0}\left[j_{s}\left(H_{S}\right) \backslash \bigcup_{0 \neq \theta \leqslant \sigma} j_{s}\left(H_{S_{\theta}}\right)\right] T^{s} \in K_{0}\left(\operatorname{Var}_{\mathbf{C}}\right)[[T]],
$$


which measures the jets of arcs through $(S, 0)$ which do not factor through any proper q.o. coordinate section $\left(S_{\theta}, 0\right), 0 \neq \theta \leqslant \sigma$. We will show that the coefficient of $T^{s}$ in the series $P(S)$ is a finite sum of classes $\left[j_{s}\left(H_{\nu}^{*}\right)\right]$. Notice that if $\tau \leqslant \sigma$ the series $P\left(S_{\tau}\right)$ is defined similarly by formula (5.2).

Proposition 5.4. (cf. Prop. 4.1 [4) We have the equality $P_{\text {geom }}^{(S, 0)}(T)=\sum_{\theta \leqslant \sigma} P\left(S_{\theta}\right)$.

Remark 5.5.

(i) The series $P\left(S_{\sigma}\right)$ takes into account those jets of arcs in $H_{S}$ which truncate to 0 . We have that $P\left(S_{\sigma}\right)=\sum_{s \geqslant 0} T^{s}=(1-T)^{-1}$.

(ii) If $\theta \leqslant \sigma$ is of codimension one then $S_{\theta}$ is a plane curve. We have that $P\left(S_{\theta}\right)=$ $\frac{\mathbf{L}-1}{1-\mathbf{L} T} \frac{T^{m}}{1-T^{m}}$, where $m$ is the multiplicity of $S_{\theta}$ (see Proposition 10.2.1 in [7]).

Recall that if $\Sigma$ is a fan then $\Sigma^{(1)}$ denotes the set of one dimensional cones of $\Sigma$ (see Section 21).

Definition 5·6. (cf. Notation 4.7 [4]) The following maps are piece-wise linear functions on the cone $\sigma$ :

$$
\left\{\begin{array}{lll}
\phi_{1}:=\operatorname{ord} \mathcal{J}_{1} & \text { and } \phi_{k}:=\quad \operatorname{ord} \mathcal{J}_{k}-\operatorname{ord} \mathcal{J}_{k-1} & \text { for } k=2, \ldots, d \\
\Psi_{1}:=0 & \text { and } \Psi_{k}:=(k-1) \operatorname{ord} \mathcal{J}_{k}-k \text { ord } \mathcal{J}_{k-1} & \text { for } k=2, \ldots, d
\end{array}\right.
$$

We define $\phi_{0}:=0$ and $\phi_{d+1}:=\infty$ by convenience. If $\rho \subset \sigma$ is a cone of dimension one, we denote by $\nu_{\rho}$ the generator of the semigroup $\rho \cap N$. We define the finite set:

$$
B(S):=\{(d, 1)\} \cup \bigcup_{k=1}^{d}\left\{\left(\Psi_{k}\left(\nu_{\rho}\right), \phi_{k}\left(\nu_{\rho}\right)\right) \mid \rho \in \cup_{i=1}^{k} \Sigma_{i}^{(1)}, \text { and } \rho \cap \stackrel{\circ}{\sigma} \neq \emptyset \text { if } k<d\right\} .
$$

This definition applies for the q.o. sections $S_{\theta}$, for $0 \leqslant \theta<\sigma$. For $\theta=\sigma$ we set $B\left(S_{\theta}\right):=$ $\{(0,1)\}$.

Definition 5·7. (cf. Def. 5.4 [4]) For $0 \leqslant k \leqslant d$ we set

$$
A_{k}:=\left\{(\nu, s) \in(\stackrel{\circ}{\sigma} \cap N) \times \mathbf{Z}_{>0} \mid \phi_{k}(\nu) \leqslant s<\phi_{k+1}(\nu)\right\} .
$$

TheOREm 5·8. (cf. Th. 7.1 [4]) If $(\nu, s) \in A_{k}$ then the jet space $j_{s}\left(H_{\nu}^{*}\right)$ is a locally closed subset of $H_{s}(S)$ isomorphic to $\{0\}$ if $k=0$ or to $\left(\mathbf{C}^{*}\right)^{k} \times \mathbf{A}_{\mathbf{C}}^{\text {sk-ord } \mathcal{J}_{k}(\nu)}$ if $1 \leqslant k \leqslant d$.

Theorem $5 \cdot 8$ is essential to prove the main results of the paper:

TheOREm 5.9. (cf. Th. 4.9 [4) Let $(S, 0)$ be an irreducible germ of q.o. hypersurface. Then there exists a polynomial $Q_{S} \in \mathbf{Z}[\mathbf{L}, T]$ determined by the lattice $M$ and the Newton polyhedra of the logarithmic jacobian ideals of $(S, 0)$ such that

$$
P(S)=Q_{S} \prod_{(a, b) \in B(S)}\left(1-\mathbf{L}^{a} T^{b}\right)^{-1} .
$$

Combining Theorem 5.9 with Proposition 5.4 we deduce the following Corollary:

Corollary 5·10. (cf. Cor. 4.10 [4]) We have that

$$
P_{\text {geom }}^{(S, 0)}(T)=\sum_{\theta \leqslant \sigma} Q_{S_{\theta}} \prod_{(a, b) \in B\left(S_{\theta}\right)}\left(1-\mathbf{L}^{a} T^{b}\right)^{-1} .
$$

The series $P_{\text {geom }}^{(S, 0)}(T)$ depends only on the logarithmic jacobian ideals and lattices $M(\theta, \zeta)$ associated to the q.o. sections $S_{\theta}$ for $0 \leqslant \theta \leqslant \sigma$ (with respect to the q.o. projection $\pi$ ). 
As an application we give a formula for the motivic volume of the arc space of a q.o. hypersurface. We refer to [5, 8, 31] for the definition of measurable sets and properties of the motivic volume.

Let $\tau$ be a strictly convex cone rational for the lattice $N$. The generating series $F_{\leftarrow \cap N}(x):=\sum_{\nu \in \leftarrow \cap N} x^{\nu}$ has a rational form

$$
F_{\tau \cap N}(x)=R_{\tau \cap N} \prod_{\rho \leqslant \tau, \operatorname{dim} \rho=1}\left(1-x^{\nu_{\rho}}\right)^{-1} \text { with } R_{\tau \cap N} \in \mathbf{Z}[\tau \cap N]
$$

(see 4 for instance). If $\tau \in \Sigma_{d}$ we denote by $\eta_{\tau}: \mathbf{Z}[\tau \cap N] \rightarrow \mathbf{Z}\left[\mathbf{L}^{ \pm 1}\right]$ the toric map given by $\eta_{\tau}\left(x^{\nu}\right)=\mathbf{L}^{- \text {ord } \mathcal{J}_{d}(\nu)}$.

Corollary 5·11. (cf. Prop. 10.1 [4]) The motivic volume of the arc space $H_{S}$ of the q.o. hypersurface $(S, 0)$ is equal to

$$
\mu\left(H_{S}\right)=(\mathbf{L}-1)^{d} \sum_{\tau \in \Sigma_{d}}^{\stackrel{\circ}{\tau} \cap \stackrel{\circ}{\sigma} \neq \emptyset} \eta_{\tau}\left(R_{\overbrace{}^{\circ} \cap N}\right) \prod_{\rho \leqslant \tau, \operatorname{dim} \rho=1}\left(1-\mathbf{L}^{-\operatorname{ord} \mathcal{J}_{d}\left(\nu_{\rho}\right)}\right)^{-1}
$$

Remark 5.12. In Section [10 we give an example of q.o. surface singularity such that all the candidate poles in Corollary $5 \cdot 10$ are actual poles.

We give a geometrical interpretation of the set of candidate poles of the series $P_{\text {geom }}^{(S, 0)}(T)$.

Definition $5 \cdot 13$. For $1 \leqslant k \leqslant d$ we denote by $\pi_{k}$ the composite of the normalization map $\bar{S}=Z^{\sigma^{\vee} \cap M} \rightarrow S$ with the toric modification of $Z_{k} \rightarrow Z^{\sigma^{\vee} \cap M}$ defined by the subdivision $\cap_{i=1}^{k} \Sigma_{i}$ of $\sigma$.

The modification $\pi_{k}$ is the minimal modification with normal source, which factors through the normalization of $S$ and the normalized blowing up of $\bar{S}$ with center $\mathcal{J}_{i}$, for $i=1, \ldots, k$. The rays $\rho$ in the fan $\cap_{i=1}^{k} \Sigma_{i}$ correspond bijectively to orbit closures of $Z_{k}$ which are of codimension one. If $\nu_{\rho}$ is the generator of the semigroup $\rho \cap N$ we denote by $E_{\nu_{\rho}}$ the irreducible component corresponding to $\rho$. We denote by $\operatorname{val}_{\nu_{\rho}}$ the divisorial valuation of the field of fractions of $\mathbf{C}\left\{\sigma^{\vee} \cap M\right\}$, which is associated to the divisor $E_{\nu_{\rho}}$. If $m \in M$ then we have that

$$
\operatorname{val}_{\nu_{\rho}}\left(X^{m}\right)=\left\langle\nu_{\rho}, m\right\rangle
$$

We have that $\stackrel{\circ}{\rho} \subset \stackrel{\circ}{\sigma}$ if and only if $E_{\nu_{\rho}}$ is a codimension one irreducible component of the exceptional fiber of $\pi_{k}^{-1}(0)$. If $1 \leqslant i \leqslant k \leqslant d$ the pull-back $\pi_{k}^{*}\left(\mathcal{J}_{i}\right)$ of $\mathcal{J}_{i}$ by $\pi_{k}$ is a locally principal monomial ideal sheaf on the toric variety $Z_{k}$ and by (5.5) we have that

$$
\operatorname{val}_{\nu_{\rho}}\left(\pi_{k}^{*}\left(\mathcal{J}_{i}\right)\right)=\operatorname{ord}_{\mathcal{J}_{i}}\left(\nu_{\rho}\right)
$$

Proposition 5·14. For $1 \leqslant k \leqslant d$ we have that

$$
\mathcal{L}_{k}:=\left(\pi_{k}^{*}\left(\mathcal{J}_{k}\right)\right)^{k-1} /\left(\pi_{k}^{*}\left(\mathcal{J}_{k-1}\right)\right)^{k} \text { and } \mathcal{Q}_{k}:=\pi_{k}^{*}\left(\mathcal{J}_{k}\right) / \pi_{k}^{*}\left(\mathcal{J}_{k-1}\right)
$$

define locally principal monomial ideal sheaves on $Z_{k}$ such that

$$
\begin{aligned}
B(S)= & \{(d, 1)\} \cup \bigcup_{k=1}^{d-1}\left\{\left(\operatorname{val}_{\nu_{\rho}}\left(\mathcal{L}_{k}\right), \operatorname{val}_{\nu_{\rho}}\left(\mathcal{Q}_{k}\right)\right) \mid E_{\nu_{\rho}} \subset \pi_{k}^{-1}(0)\right\} \\
& \cup\left\{\left(\operatorname{val}_{\nu_{\rho}}\left(\mathcal{L}_{d}\right), \operatorname{val}_{\nu_{\rho}}\left(\mathcal{Q}_{d}\right)\right) \mid \rho \in \cap_{i=1}^{d} \Sigma_{i}, \operatorname{dim} \rho=1\right\} .
\end{aligned}
$$


6. Combinatorial convexity properties of the logarithmic jacobian ideals

In this section we give a series of results on the properties of the support functions of the logarithmic jacobian ideals associated to a q.o. hypersurface germ $(S, 0)$, which are used in Sections 7 and 8

Notation $6 \cdot 1$. If $\nu \in \sigma \cap N$ we denote by $\leqslant{ }_{\nu}$ the partial order on $M$ defined by $\lambda_{\leqslant} \lambda^{\prime}$ if $\langle\nu, \lambda\rangle \leqslant\left\langle\nu, \lambda^{\prime}\right\rangle$.

Remark 6.2. The logarithmic jacobian ideals $\overline{\mathcal{J}}_{1}, \ldots, \overline{\mathcal{J}}_{d}$ of the normalization $(\bar{S}, 0)$, which are studied in [4, are different than those of $(S, 0)$ in general. Recall that we have $\bar{S}=Z^{\sigma^{\vee} \cap M}$. If $v_{1}, \ldots, v_{n}$ are the minimal sequence of generators of the semigroup

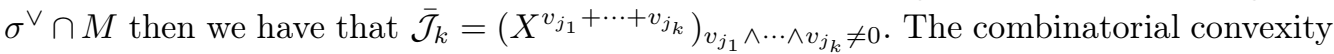
properties of the support functions, ord $\overline{\mathcal{J}}_{k}$ for $k=1, \ldots, d$, are simpler in the toric case. Given $\nu \in \stackrel{\circ}{\sigma} \cap N$, up to relabeling, we can assume for simplicity that $v_{1} \leqslant \nu \cdots \leqslant{ }_{\nu} v_{n}$. We define inductively $i_{1}:=1$ and $i_{k}:=\min \left\{i \mid v_{i_{1}} \wedge \cdots \wedge v_{i_{k-1}} \wedge v_{i} \neq 0\right\}$ for $k=2, \ldots, d$. Then we have that ord $\overline{\mathcal{J}}_{k}(\nu):=\sum_{l=1}^{k}\left\langle\nu, v_{i_{l}}\right\rangle$ and ord ${\overline{\mathcal{J}_{k+1}}}(\nu)-\operatorname{ord}_{\overline{\mathcal{J}}_{k}}(\nu)=\left\langle\nu, v_{i_{l}}\right\rangle$.

The following example shows that the q.o. case is not as simple as the toric case.

Example 6.3. Consider a q.o. branch with characteristic exponents $\lambda_{1}=(1 / 2,1 / 2,0)$ and $\lambda_{2}=(1 / 2,1 / 2,1 / 4)$. The points $\nu_{1}=(4,2,8)$ and $\nu_{2}=(4,2,4)$ belong to the lattice $N$. It is easy to check that ord $\mathcal{J}_{2}\left(\nu_{1}\right)=\left\langle\nu_{1}, e_{2}+\lambda_{1}\right\rangle$, ord $\mathcal{J}_{2}\left(\nu_{2}\right)=\left\langle\nu_{2}, e_{2}+\right.$ $\left.\lambda_{1}\right\rangle$, ord $\mathcal{J}_{3}\left(\nu_{1}\right)=\left\langle\nu_{1}, e_{1}+e_{2}+\lambda_{2}\right\rangle$ and $\operatorname{ord}_{\mathcal{J}_{3}}\left(\nu_{2}\right)=\left\langle\nu_{2}, e_{2}+\lambda_{1}+e_{3}\right\rangle$. Then we get $\left(\operatorname{ord}_{\mathcal{J}_{3}}-\operatorname{ord}_{\mathcal{J}_{2}}\right)\left(\nu_{1}\right)=\left\langle\nu_{1}, \lambda_{2}-\lambda_{1}+e_{3}\right\rangle$ while $\left(\operatorname{ord}_{\mathcal{J}_{3}}-\operatorname{ord} \mathcal{J}_{2}\right)\left(\nu_{2}\right)=\left\langle\nu_{2}, e_{3}\right\rangle$.

Notation $6 \cdot 4$. We fix a partial order on the set $\left\{e_{1}, \ldots, e_{d+g}\right\}$ by

$$
e_{i_{1}} \leqslant \nu \ldots \leqslant \nu e_{i_{d+g}}
$$

in such a way that if $\left\langle\nu, e_{i_{j}}\right\rangle=\left\langle\nu, e_{i_{k}}\right\rangle$ for $1 \leqslant i_{j} \leqslant d$ and $d+1 \leqslant i_{k} \leqslant d+g$, then $j<k$.

By Lemma $3 \cdot 1$ we have the inequalities

$$
\left\langle\nu, \lambda_{1}\right\rangle<\left\langle\nu, \lambda_{2}\right\rangle<\cdots<\left\langle\nu, \lambda_{g}\right\rangle
$$

In the following Proposition we use the convention $\langle\nu, \infty\rangle=\infty>r$, for $r \in \mathbf{R}$ (see Notations [5.1).

Proposition 6.5. (cf. Prop. 5.1 [4] Let $\nu \in \stackrel{\circ}{\sigma} \cap N$. With respect to the order (6.1), the vector $\nu$ defines a sequence $1 \leqslant j_{1}^{(k)}<\cdots<j_{k}^{(k)} \leqslant d+$ g such that $w_{k}:=e_{j_{1}^{(k)}}+\cdots+e_{j_{k}^{(k)}} \in$ $\mathcal{J}_{k}$ and $\operatorname{ord}_{\mathcal{J}_{k}}(\nu)=\left\langle\nu, w_{k}\right\rangle$. We set $w_{1}=e_{j_{1}^{(1)}}:=e_{i_{1}}$. Suppose that $w_{k}=e_{j_{1}^{(k)}}+\cdots+e_{j_{r}^{(k)}}$ is already defined. Set:

(i) $\ell_{k}(\nu):=\operatorname{span}_{\mathbf{Q}}\left\{e_{j_{1}^{(k)}}, \ldots, e_{j_{k}^{(k)}}\right\}$.

(ii) $n(k):=\left\{\begin{array}{l}0 \text { if }\left\{e_{j_{1}^{(k)}}, \ldots, e_{j_{k}^{(k)}}\right\} \backslash\left\{e_{1}, \ldots, e_{d}\right\}=\emptyset, \\ n \text { if }\left\{e_{j_{1}^{(k)}}, \ldots, e_{j_{k}^{(k)}}\right\} \backslash\left\{e_{1}, \ldots, e_{d}\right\}=\left\{\lambda_{n}\right\} .\end{array}\right.$

(iii) $t(k):=\min \left\{1 \leqslant j \leqslant g+1 \mid \lambda_{j} \notin \ell_{k}(\nu)\right\}$.

(iv) $m(k):=\left\{\begin{array}{l}0 \text { if }\left(\left\{e_{1}, \ldots, e_{d}\right\} \cap \ell_{k}(\nu)\right) \backslash\left\{e_{j_{1}^{(k)}}, \ldots, e_{j_{k}^{(k)}}\right\}=\emptyset \\ m \text { if }\left(\left\{e_{1}, \ldots, e_{d}\right\} \cap \ell_{k}(\nu)\right) \backslash\left\{e_{j_{1}^{(k)}}, \ldots, e_{j_{k}^{(k)}}\right\}=\left\{e_{m}\right\} .\end{array}\right.$

(v) $i(k):=\min \left\{1 \leqslant i \leqslant d+g \mid w_{k}+e_{i} \in \mathcal{J}_{k+1}\right\}$, for $k=1, \ldots, d-1$. 


$$
\begin{gathered}
\text { Set } a_{k+1}:=w_{k}+e_{i(k)} \text { and } b_{k+1}:=w_{k}-\lambda_{n(k)}+\lambda_{t(k)}+e_{m(k)} \text {. Then we have that: } \\
\qquad w_{k+1}:= \begin{cases}a_{k+1} & \text { if }\left\langle\nu, a_{k+1}\right\rangle \leqslant\left\langle\nu, b_{k+1}\right\rangle, \\
b_{k+1} & \text { otherwise. }\end{cases}
\end{gathered}
$$

Proof. We prove it by induction on $k$. For $k=1$ the assertion holds, since $\left\langle\nu, e_{i_{1}}\right\rangle=$ $\min \left\{\left\langle\nu, e_{i}\right\rangle \mid 1 \leqslant i \leqslant d+1\right\}$. We suppose that the statement is true for $k$ and we prove it for $k+1$. By induction hypothesis we have $w_{k}=e_{j_{1}^{(k)}}+\cdots+e_{j_{k}^{(k)}} \in \mathcal{J}_{k}$ and $\operatorname{ord}_{\mathcal{J}_{k}}(\nu)=\left\langle\nu, w_{k}\right\rangle$. If $n(k)=0$ the argument coincides with the proof of Proposition 5.1 in [4. We assume that $n(k) \neq 0$ and that there exists $w_{k+1}^{\prime} \in \mathcal{J}_{k+1}$ different from $w_{k+1}$ and such that

$$
\left\langle\nu, w_{k+1}^{\prime}\right\rangle<\left\langle\nu, w_{k+1}\right\rangle
$$

Assertion 6.6. If the vector $e_{j}$ with $1 \leqslant j \leqslant d$ and $j \neq m(k)$ appears in the expansion of $w_{k+1}^{\prime}$ as sum of $k+1$ linearly independent elements of $\left\{e_{1}, \ldots, e_{d+g}\right\}$, then $e_{j}$ appears in the expansion of $w_{k+1}$.

Proof of the assertion. If $e_{j}$ does not appear in the expansion of $w_{k+1}$, then $w_{k+1}^{\prime}-e_{j}$ belongs to $\mathcal{J}_{k}$ and we deduce $\left\langle\nu, w_{k}\right\rangle \leqslant\left\langle\nu, w_{k+1}^{\prime}-e_{j}\right\rangle\left\langle\left\langle\nu, w_{k+1}-e_{j}\right\rangle\right.$, where the first inequality follows by the induction hypothesis and the second by (6.4). Then we get $\left\langle\nu, w_{k}+e_{j}\right\rangle<\left\langle\nu, w_{k+1}\right\rangle$ and since $j \neq m(k)$ the vector $w_{k}+e_{j}$ belongs to $\mathcal{J}_{k+1}$, but this is in contradiction with the choice of $w_{k+1}$ in the algorithm, hence the assertion holds.

Now we distinguish various cases:

(i) If $m(k)=0$, then we obtain $w_{k+1}=w_{k}+e_{i(k)}$ by definition. By the Assertion there is an integer $1 \leqslant r \leqslant g$ such that $w_{k+1}^{\prime}-w_{k+1}=\lambda_{r}-\lambda_{n(k)}$. By (6.2) and (6.4) we deduce that $r<n(k)$, but then $w_{k+1}^{\prime}-e_{i(k)}=w_{k}-\lambda_{n(k)}+\lambda_{r} \in \mathcal{J}_{k}$ and $\left\langle\nu, w_{k+1}^{\prime}-e_{i(k)}\right\rangle\left\langle\left\langle\nu, w_{k}\right\rangle\right.$, This is a contradiction with the induction hypothesis.

(ii) If $m(k) \neq 0$ and $w_{k+1}=w_{k}+e_{i(k)}$ and if $e_{m(k)}$ does not appear in the expansion of $w_{k+1}^{\prime}$ we apply the argument of case (i) to get a contradiction.

(iii) If $m(k) \neq 0, w_{k+1}=w_{k}+e_{i(k)}$ and if $e_{m(k)}$ appears in the expansion of $w_{k+1}^{\prime}$ then by the Assertion we have that $w_{k+1}=w+\lambda_{n(k)}+e_{i(k)}$ and $w_{k+1}^{\prime}=w+\lambda_{r}+e_{m(k)}$, where $w=w_{k}-\lambda_{n(k)} \in \mathcal{J}_{k-1}$. By definition $e_{m(k)} \in \ell_{k}(\nu)$, hence $\lambda_{r}$ does not belong to $\ell_{k}(\nu)$ since $w_{k+1}^{\prime} \in \mathcal{J}_{k+1}$. By definition of $t(k)$ we deduce that $r \geqslant t(k)>$ $n(k)$. Then it follows that $\left\langle\nu, w_{k+1}^{\prime}\right\rangle \geqslant\left\langle\nu, w_{k}-\lambda_{n(k)}+\lambda_{t(k)}+e_{m(k)}\right\rangle \geqslant\left\langle\nu, w_{k+1}\right\rangle$, which contradicts (6.4).

(iv) If $m(k) \neq 0$ and $w_{k+1}=w_{k}-\lambda_{n(k)}+\lambda_{t(k)}+e_{m(k)}$ the assertion implies that $e_{m(k)}$ appears in the expansion of $w_{k+1}^{\prime} \in \mathcal{J}_{k+1}$. We deduce that $w_{k+1}=w+\lambda_{t(k)}+e_{m(k)}$ and $w_{k+1}^{\prime}=w+\lambda_{r}+e_{m(k)}$. Formula (6.4) implies that $r<t(k)$ which is a contradiction with the definition of $t(k)$.

The two choices appearing in (6.3) occur (see Example 6.3).

Remark 6.7. Notice that $m(k)>0$ implies $n(k)>0$ and $\left\langle\nu, \lambda_{n(k)}\right\rangle\left\langle\left\langle\nu, e_{m(k)}\right\rangle\right.$ (otherwise $w_{k}^{\prime}=w_{k}-\lambda_{n(k)}+e_{m(k)} \in \mathcal{J}_{k}$ would verify that $\left.\left\langle\nu, w_{k}^{\prime}\right\rangle<\operatorname{ord}_{\mathcal{J}_{k}}(\nu)\right)$. 
Lemma 6•8. (cf. Lemma 5.3 [4]) For all $\nu \in \stackrel{\circ}{\mathrm{\sigma}} \cap N$ we have that

$$
\phi_{1}(\nu) \leqslant \phi_{2}(\nu) \leqslant \cdots \leqslant \phi_{d}(\nu) .
$$

Proof. The assertion is equivalent to the inequality $\left\langle\nu, w_{k}-w_{k-1}\right\rangle \leqslant\left\langle\nu, w_{k+1}-w_{k}\right\rangle$ for $1 \leqslant k<d$, where $w_{k-1}, w_{k}$ and $w_{k+1}$ are defined by the algorithm and $w_{0}:=0$. We distinguish the cases:

(i) If $\phi_{k}(\nu)=\left\langle\nu, e_{i(k-1)}\right\rangle$ and $\phi_{k+1}(\nu)=\left\langle\nu, e_{i(k)}\right\rangle$ the result follows by definition of $e_{i(k-1)}$ in Proposition 6.5.

(ii) If $\phi_{k}(\nu)=\left\langle\nu, e_{i(k-1)}\right\rangle$ and $\phi_{k+1}(\nu)=\left\langle\nu, \lambda_{t(k)}-\lambda_{n(k)}+e_{m(k)}\right\rangle$, we get $w_{k}=$ $w_{k-1}+e_{i(k-1)}$ and $w_{k+1}=w_{k}-\lambda_{n(k)}+\lambda_{t(k)}+e_{m(k)}$. We distinguish two subcases below:

(ii.1) If $e_{i(k-1)}=\lambda_{n(k)}$ we get $\left\langle\nu, e_{m(k)}\right\rangle>\left\langle\nu, \lambda_{n(k)}\right\rangle$ by the definition of the algorithm hence we deduce $\phi_{k}(\nu)=\left\langle\nu, \lambda_{n(k)}\right\rangle\left\langle\left\langle\nu, \lambda_{t(k)}-\lambda_{n(k)}+e_{m(k)}\right\rangle=\right.$ $\phi_{k+1}(\nu)$ since $t(k)>n(k)$.

(ii.2) If $e_{i(k-1)} \neq \lambda_{n(k)}$ we obtain $1 \leqslant i(k-1) \leqslant d$ and $\phi_{k}(\nu)=\left\langle\nu, e_{i(k-1)}\right\rangle \leqslant$ $\left\langle\nu, e_{m(k)}\right\rangle<\left\langle\nu, \lambda_{t(k)}-\lambda_{n(k)}+e_{m(k)}\right\rangle=\phi_{k+1}(\nu)$.

(iii) If $\phi_{k}(\nu)=\left\langle\nu, \lambda_{t(k-1)}-\lambda_{n(k-1)}+e_{m(k-1)}\right\rangle$ and $\phi_{k+1}(\nu)=\left\langle\nu, e_{i(k)}\right\rangle$ then we get $w_{k}=w_{k-1}-\lambda_{n(k-1)}+\lambda_{t(k-1)}+e_{m(k-1)}$ and $w_{k+1}=w_{k}+e_{i(k)}$. Since the vector $e_{i(k)}$ is not a characteristic exponent we deduce that $w_{k-1}+e_{i(k)} \in \mathcal{J}_{k}$. Then we get the inequalities $\left\langle\nu, w_{k}\right\rangle \leqslant\left\langle\nu, w_{k-1}+e_{i(k)}\right\rangle$, and it follows that: $\phi_{k}(\nu)=$ $\left\langle\nu, \lambda_{t(k-1)}-\lambda_{n(k-1)}+e_{m(k-1)}\right\rangle \leqslant\left\langle\nu, e_{i(k)}\right\rangle=\phi_{k+1}(\nu)$.

(iv) If $\phi_{k}(\nu)=\left\langle\nu, \lambda_{t(k-1)}-\lambda_{n(k-1)}+e_{m(k-1)}\right\rangle$ and $\phi_{k+1}(\nu)=\left\langle\nu, \lambda_{t(k)}-\lambda_{n(k)}+e_{m(k)}\right\rangle$ we obtain $w_{k}=w_{k-1}-\lambda_{n(k-1)}+\lambda_{t(k-1)}+e_{m(k-1)}$ and $w_{k+1}=w_{k}-\lambda_{n(k)}+$ $\lambda_{t(k)}+e_{m(k)}$. Since the vector $w_{k-1}+e_{m(k)}$ belongs to $\mathcal{J}_{k}$ we deduce $\left\langle\nu, w_{k}\right\rangle<$ $\left\langle\nu, w_{k-1}+e_{m(k)}\right\rangle$. We get $\phi_{k}(\nu)=\left\langle\nu, \lambda_{t(k-1)}-\lambda_{n(k-1)}+e_{m(k-1)}\right\rangle\left\langle\left\langle\nu, e_{m(k)}\right\rangle<\right.$ $\left\langle\nu, \lambda_{t(k)}-\lambda_{n(k)}+e_{m(k)}\right\rangle=\phi_{k+1}(\nu)$.

Definition 6.9. If $(\nu, s) \in \stackrel{\circ}{\sigma} \times \mathbf{Z}_{\geqslant 0}$ there is a unique integer $0 \leqslant k \leqslant d$ such that $(\nu, s) \in A_{k}$. We set

$$
\ell_{\nu}^{s}:=\operatorname{span}_{\mathbf{Q}}\left\{e_{j} \mid 1 \leqslant j<d+t(k),\left\langle\nu, e_{j}\right\rangle \leqslant s\right\} .
$$

(cf. Definition [5.7 Proposition 6.5 and Lemma 6.8).

Lemma 6-10. (cf. Lemma 5.7 [4]) If $(\nu, s) \in A_{k}$ and $w_{k}^{\prime}=e_{r_{1}}+\cdots+e_{r_{k}} \in \mathcal{J}_{k}$ is a vector such that $\operatorname{ord}_{\mathcal{J}_{k}}(\nu)=\left\langle\nu, w_{k}^{\prime}\right\rangle$, then we have

$$
\ell_{\nu}^{s}=\ell_{k}(\nu)=\operatorname{span}_{\mathbf{Q}}\left\{e_{r_{1}}, \ldots, e_{r_{k}}\right\} .
$$

In addition, if $1 \leqslant j<n(k)$ and $\lambda_{j}=\sum_{i=1}^{d} \lambda_{j, i} e_{i}$ then $\lambda_{j, i} \neq 0$ implies that $\left\langle\nu, e_{i}\right\rangle \leqslant$ $\left\langle\nu, \lambda_{j}\right\rangle \leqslant s$.

Proof. Let $w_{k}=\sum_{r=1}^{k} e_{j_{r}(k)}$ be the vector defined by Proposition 6.5 We denote by $\ell=\ell_{k}(\nu)$ (resp. by $\ell^{\prime}$ ) the linear subspace of $M_{\mathbf{Q}}$ spanned by the vectors in the expansion of $w_{k}$ (resp. $\left.w_{k}^{\prime}\right)$. We prove first that $\ell=\ell^{\prime}$. If $\ell^{\prime}$ and $\ell$ are distinct we verify that $\phi_{k}(\nu)=\phi_{k+1}(\nu)$. Suppose that there exists a vector $e_{j_{0}}$ appearing in the expansion of $w_{k}^{\prime}$ and such that $e_{j_{0}} \notin \ell$. We distinguish two cases:

(i) If $1 \leqslant j_{0} \leqslant d$ then the vector $w_{k+1}^{\prime}:=w_{k}+e_{j_{0}}$ belongs to $\mathcal{J}_{k+1}$. We get $\operatorname{ord}_{\mathcal{J}_{k+1}}(\nu) \leqslant\left\langle\nu, w_{k+1}^{\prime}\right\rangle=\operatorname{ord}_{\mathcal{J}_{k}}(\nu)+\left\langle\nu, e_{j_{0}}\right\rangle$ hence $\phi_{k+1}(\nu) \leqslant\left\langle\nu, e_{j_{0}}\right\rangle$. If $\left.\left\langle\nu, e_{j_{0}}\right\rangle\right\rangle$ 
$\phi_{k}(\nu)$ then the vector $w_{k-1}^{\prime}:=w_{k}^{\prime}-e_{j_{0}}$ belongs to $\mathcal{J}_{k-1}$ and we find the contra$\operatorname{diction}\left\langle\nu, w_{k-1}^{\prime}\right\rangle=\operatorname{ord}_{\mathcal{J}_{k}}(\nu)-\left\langle\nu, e_{j_{0}}\right\rangle\left\langle\operatorname{ord}_{\mathcal{J}_{k}}(\nu)-\phi_{k}(\nu)=\operatorname{ord}_{\mathcal{J}_{k-1}}(\nu)\right.$. Hence we obtain $\phi_{k}(\nu) \geqslant\left\langle\nu, e_{j_{0}}\right\rangle$ thus $\phi_{k}(\nu)=\phi_{k+1}(\nu)$ holds by Lemma $6 \cdot 8$.

(ii) If for any $1 \leqslant j \leqslant d$, with $e_{j}$ appearing in the expansion of $w_{k}^{\prime}$ then $e_{j} \in \ell$, then we get $w_{k}=w+e_{r}$ and $w_{k}^{\prime}=w+\lambda_{n^{\prime}}$, for $1 \leqslant r \leqslant d+g$ and $1 \leqslant n^{\prime} \leqslant g$. Then $\left\langle\nu, \lambda_{n^{\prime}}\right\rangle=\left\langle\nu, e_{r}\right\rangle$ and by (6.2) the vector $e_{r}$ can not be a characteristic exponent. Thus $n(k)=0$ and we get $w_{k+1}=w_{k}+\lambda_{n^{\prime}}$. We obtain $\phi_{k+1}(\nu)=\left\langle\nu, \lambda_{n^{\prime}}\right\rangle=$ $\left\langle\nu, e_{j}\right\rangle \leqslant \phi_{k}(\nu)$ thus $\phi_{k}(\nu)=\phi_{k+1}(\nu)$ holds by Lemma 6.8

We obtain that $\ell \subset \ell_{\nu}^{s}$ by checking that $\left\langle\nu, e_{j_{r}(k)}\right\rangle \leqslant s$ for $r=1, \ldots, k$. If $k=1$ the inequality is trivial. If $k>1$ and $e_{j_{r}(k)}$ is a vector appearing in the expansion of $w_{k}$ then there are two possibilities.

(i) If there is $1 \leqslant l \leqslant k-1$ such that $w_{l+1}=w_{l}+e_{j_{r}^{(k)}}$ then we get $\phi_{l+1}(\nu)=\left\langle\nu, e_{j_{r}^{(k)}}\right\rangle$. By Lemma $6 \cdot 8$ we deduce $\left\langle\nu, e_{j_{r}^{(k)}}\right\rangle \leqslant s$.

(ii) If there is $1 \leqslant l \leqslant k-1$ such that $w_{l+1}=w_{l}-\lambda_{n(l)}+\lambda_{t(l)}+e_{m(l)}$, with either $\lambda_{t(l)}=e_{j_{r}^{(k)}}$ or $e_{m(l)}=e_{j_{r}^{(k)}}$ then in both cases we get $\phi_{l+1}(\nu) \geqslant\left\langle\nu, e_{j_{r}^{(k)}}\right\rangle$, since $\left\langle\nu, \lambda_{n(l)}\right\rangle<\left\langle\nu, e_{m(l)}\right\rangle$ and $n(l)<t(l)$.

We prove that $\ell_{\nu}^{s} \subset \ell$. If $\left\langle\nu, e_{j}\right\rangle \leqslant s$ for $1 \leqslant j \leqslant d$ and if $e_{j} \notin \ell$ then $\widetilde{w}_{k+1}:=$ $w_{k}+e$ belongs to $\mathcal{J}_{k+1}$; we deduce that $\phi_{k+1}(\nu) \leqslant\left\langle\nu, \widetilde{w}_{k+1}-w_{k}\right\rangle \leqslant s$ contradicting the hypothesis. We have also shown that $\lambda_{n(k)} \in \ell$ hence if $n(k)<j<t(k)$ then $\lambda_{j} \in \ell$ by definition of $t(k)$. If $1 \leqslant j<n(k)$ then $\left\langle\nu, \lambda_{j}\right\rangle\left\langle\left\langle\nu, \lambda_{n(k)}\right\rangle \leqslant s\right.$ by (6.2) and it is easy to see from the algorithm in Proposition 6.5 that $\lambda_{j}$ belongs to $\operatorname{span}_{\mathbf{Q}}\left\{e_{i} \mid 1 \leqslant i \leqslant\right.$ $\left.d,\left\langle\nu, e_{i}\right\rangle \leqslant s\right\} \subset \ell$. It follows that $\ell_{\nu}^{s} \subset \ell$.

For the last assertion notice that if $\lambda_{j, i} \neq 0$ and if $\left\langle\nu, e_{i}\right\rangle>\left\langle\nu, \lambda_{j}\right\rangle$ then we get a contradiction since $w_{k}^{\prime}:=\lambda_{j}+\sum_{r=1, \ldots, d, r \neq i, m(k)}^{\left\langle\nu, e_{r}\right\rangle \leqslant s} e_{r}$ belongs to $\mathcal{J}_{k}$ and verifies that $\left\langle\nu, w_{k}^{\prime}\right\rangle<\left\langle\nu, w_{k}\right\rangle$.

Lemma 6·11. If $(\nu, s) \in A_{k}$ we have the following inequalities

(i) $s<\left\langle\nu, \lambda_{t(k)}\right\rangle$ if $n(k)=0$ or if $n(k), m(k) \neq 0$ and $\left\langle\nu, e_{m(k)}\right\rangle \leqslant s$.

(ii) $s<\left\langle\nu, e_{m(k)}+\lambda_{t(k)}-\lambda_{n(k)}\right\rangle$ if $n(k) \neq 0$.

Proof. If $n(k)=0$ then the vector $\lambda_{t(k)}+e_{j_{1}^{(k)}}+\cdots+e_{j_{1}^{(k)}}$ belongs to $\mathcal{J}_{k+1}$ hence $\operatorname{ord}_{\mathcal{J}_{k+1}}(\nu) \leqslant\left\langle\nu, \lambda_{t(k)}\right\rangle+\operatorname{ord}_{\mathcal{J}_{k}}(\nu)$. We deduce from this that $\phi_{k+1}(\nu) \leqslant\left\langle\nu, \lambda_{t(k)}\right\rangle$. If $n(k), m(k) \neq 0$ then we get $\phi_{k+1}(\nu) \leqslant\left\langle\nu, e_{m(k)}+\lambda_{t(k)}-\lambda_{n(k)}\right\rangle$ by proof of Lemma 6.8 This implies that $s<\left\langle\nu, \lambda_{t(k)}\right\rangle$ since $\left\langle\nu, e_{m(k)}-\lambda_{n(k)}\right\rangle>0$ by Remark 6.7.

Remark 6.12. If $(\nu, s) \in A_{k}, n(k), m(k) \neq 0$ it may happen that $\left\langle\nu, \lambda_{t(k)}\right\rangle \leqslant s$. For instance, consider a q.o. branch with characteristic exponents $\lambda_{1}:=\left(\frac{1}{2}, \frac{1}{8}, 0\right)$ and $\lambda_{2}:=$ $\left(\frac{1}{2}, \frac{1}{8}, \frac{1}{18}\right)$. If $\nu=(12,16,18) \in N$ then we get $\phi_{1}(\nu)=8, \phi_{2}(\nu)=12$ and $\phi_{3}(\nu)=17$. If $12 \leqslant s<17$ then we obtain $(\nu, s) \in A_{2}, n(2)=1, m(2)=2, t(2)=2$ and $\left\langle\nu, \lambda_{2}\right\rangle=9 \leqslant s$.

Definition 6.13.

(i) If $\eta=\eta_{1} e_{1}+\cdots+\eta_{d} e_{d} \in M$ we denote by $\ell(\eta)=\operatorname{span}_{\mathbf{Q}}\left\{e_{i} \mid \eta_{i} \neq 0\right\}$ the smallest coordinate subspace containing $\eta$.

(ii) With respect to the fixed vector $\nu \in \stackrel{\circ}{\sigma} \cap N$, we set $e_{q_{\eta}}=\max _{\leqslant_{\nu}}\left\{\left\{e_{1}, \ldots, e_{d}\right\} \cap \ell(\eta)\right\}$.

Remark 6.14. It is easy to see from the algorithm in Proposition 6.5 that if $1 \leqslant j<n(k)$ then $\ell\left(\lambda_{j}\right) \subset \ell_{\nu}^{s}$ and if $e_{m(k)} \neq \infty$ then $q_{\lambda_{n(k)}}=m(k)$. 
Definition 6.15. Let $(\nu, s) \in A_{k}$ for some $k, s>0$. With the notations of Proposition 6.5 we define the integer $p(k)$ by

$$
p(k):= \begin{cases}\max \left\{0 \leqslant j \leqslant g \mid\left\langle\nu, \lambda_{j}\right\rangle \leqslant s\right\} & \text { if } n(k)=0, \\ \max \left(\{n(k)\} \cup\left\{n(k)<j<t(k) \mid\left\langle\nu, e_{m(k)}-\lambda_{n(k)}+\lambda_{j}\right\rangle \leqslant s\right\}\right) & \text { if } n(k)>0 .\end{cases}
$$

Remark 6·16. Notice that $\left\langle\nu, \lambda_{p(k)}\right\rangle \leqslant s$.

Lemma 6-17. If $(\nu, s) \in A_{k}$ for $1 \leqslant k \leqslant d$ and $1 \leqslant j<p(k)$ the following inequality holds

$$
\left\langle\nu, \lambda_{p(k)}-\lambda_{j}\right\rangle \leqslant s-\left\langle\nu, e_{q_{\lambda_{j}}}\right\rangle .
$$

If in addition $n(k)>0$ and $m(k) \neq 0$ we have

$$
\left\langle\nu, e_{q_{\lambda_{j}}}\right\rangle \leqslant\left\langle\nu, e_{m(k)}-\lambda_{n(k)}+\lambda_{j}\right\rangle .
$$

Proof. Suppose first that $\left\langle\nu, \lambda_{j}\right\rangle \geqslant\left\langle\nu, e_{q_{\lambda_{j}}}\right\rangle$ for some $1 \leqslant j \leqslant p(k)$. It follows that (6.6) holds by Remark [6.7 We deduce also that (6.5) holds since $\left\langle\nu, \lambda_{p(k)}\right\rangle \leqslant s$ hence $\left\langle\nu, \lambda_{p(k)}-\lambda_{j}\right\rangle \leqslant s-\left\langle\nu, \lambda_{j}\right\rangle \leqslant s-\left\langle\nu, e_{q_{\lambda_{j}}}\right\rangle$.

We deal first with the case $1 \leqslant j<n(k)$. If $\left\langle\nu, \lambda_{j}\right\rangle<\left\langle\nu, e_{q_{\lambda_{j}}}\right\rangle$ then the set $\{1 \leqslant r \leqslant$ $j \mid \lambda_{r}=\lambda_{n\left(k^{\prime}\right)}$ for $\left.1 \leqslant k^{\prime} \leqslant k\right\}$ is non empty and $n(k)>0$. We introduce the terms $\lambda_{r_{0}}, \ldots, \lambda_{r_{h}}=\lambda_{n(k)}$, which are the characteristic exponents appearing in the expansion of the terms $w_{k_{0}}, \ldots, w_{k_{h}}$ defined in Proposition 6.5 by the vector $\nu$ for suitable integers $k_{0}, \ldots, k_{h}$. First we set

$r_{0}:=\max \left\{1 \leqslant r \leqslant j \mid \lambda_{r}=\lambda_{n\left(k^{\prime}\right)}\right.$ for $\left.1 \leqslant k^{\prime}<k\right\}, k_{0}:=\min \left\{1 \leqslant k^{\prime}<k \mid \lambda_{r_{0}}=\lambda_{n\left(k^{\prime}\right)}\right\}$, and then inductively $r_{i}:=\min \left\{r_{i-1}<r \leqslant n(k) \mid \lambda_{r}=\lambda_{n\left(k^{\prime}\right)}, k_{i-1}<k^{\prime} \leqslant k\right\}$ and $k_{i}:=\min \left\{k_{i-1} \leqslant k^{\prime} \leqslant k \mid \lambda_{r_{i}}=\lambda_{n\left(k^{\prime}\right)}\right\}$. After finitely many steps we have an integer $h$ such that $k_{h} \leqslant k$ and $r_{h}=n(k)$, and the process stops.

It is easy to check that $r_{0} \leqslant j<r_{1}\left\langle\nu, \lambda_{r_{0}}\right\rangle \leqslant\left\langle\nu, \lambda_{j}\right\rangle\left\langle\left\langle\nu, \lambda_{r_{1}}\right\rangle\right.$. Notice that $e_{q_{\lambda_{r_{l}}}}=$ $e_{m\left(k_{l}\right)}$ for $l=0, \ldots, h-1$ and if $m(k) \neq 0$ we have also that $e_{{\lambda_{\lambda_{h}}}}=e_{m\left(k_{h}\right)}=e_{m(k)}$.

Remark that the definition of $w_{k_{1}}, \ldots, w_{k_{h}}$ in Proposition 6.5] involves the choice $b_{k_{i}}$ in (6.3), i.e., we have:

$$
\phi_{k_{l-1}}(\nu)=\left\langle\nu, \lambda_{r_{l}}-\lambda_{r_{l-1}}+e_{{\lambda_{\lambda_{l}-1}}}\right\rangle<\left\langle\nu, e_{q_{\lambda_{r_{l}}}}\right\rangle \text { for } l=1, \ldots, h-1 .
$$

and similarly by Lemma $6 \cdot 8$ and Definition $5 \cdot 7$

$$
\phi_{k_{h}}(\nu)=\left\langle\nu, \lambda_{r_{h}}-\lambda_{r_{h-1}}+e_{q_{\lambda_{h-1}}}\right\rangle \leqslant \begin{cases}s & \text { if } m(k)=0, \\ \left\langle\nu, e_{m(k)}\right\rangle & \text { if } m(k) \neq 0 .\end{cases}
$$

Summing the inequalities in (6.7) for $l=1, \ldots, h-1$ with (6.8) provides the inequality

$$
\left\langle\nu, \lambda_{r_{h}}-\lambda_{r_{0}}+e_{q_{\lambda_{r_{0}}}}\right\rangle \leqslant \begin{cases}s & \text { if } m(k)=0, \\ \left\langle\nu, e_{m(k)}\right\rangle & \text { if } m(k) \neq 0 .\end{cases}
$$

Now we distinguish two cases:

(i) If $\ell\left(\lambda_{r_{0}}\right)=\ell\left(\lambda_{j}\right)$ then $q_{\lambda_{r_{0}}}=q_{\lambda_{j}}$ by definition. Then the inequality (6.6) hold by (6.9) since $\left\langle\nu, \lambda_{r_{0}}\right\rangle \leqslant\left\langle\nu, \lambda_{j}\right\rangle$. Adding (6.9) with the inequality

$$
\left\langle\nu, \lambda_{p(k)}-\lambda_{n(k)}+e_{m(k)}\right\rangle \leqslant s
$$

shows that (6.5) holds. 
(ii) Otherwise $\ell\left(\lambda_{r_{0}}\right) \varsubsetneqq \ell\left(\lambda_{j}\right)$ and we have that $\lambda_{j} \neq \lambda_{n\left(k^{\prime}\right)}$ for $k_{0}<k^{\prime}<k$. The characteristic exponent $\lambda_{j}$ is not chosen at any step of the algorithm in Proposition 6.5. that is,

$$
\left\langle\nu, e_{q_{\lambda_{j}}}\right\rangle<\left\langle\nu, \lambda_{j}-\lambda_{r_{0}}+e_{q_{r_{0}}}\right\rangle .
$$

Then we check that (6.6) holds by adding the inequalities (6.11) and (6.9). The same happens for (6.5) by adding (6.11), (6.9) and (6.10). It remains to prove that (6.5) and (6.6) hold for $n(k) \leqslant j<p(k)$. In this case $e_{q_{\lambda_{j}}}=e_{m(k)}$ and the inequalities hold trivially by the definition of $p(k)$ since $\left\langle\nu, \lambda_{n(k)}\right\rangle \leqslant\left\langle\nu, \lambda_{j}\right\rangle$.

\section{The jet space $j_{s}\left(H_{S, \nu}^{*}\right)$}

Notation 7.1. In this section we fix $(\nu, s) \in A_{k}$ for some $0 \leqslant k \leqslant d$ and we simplify our notations. Let $w_{k}=e_{j_{1}^{(k)}}+\cdots+e_{j_{k}^{(k)}}$ be the vector defined by $\nu$ in Proposition 6.5 We relabel the vectors in $\left\{e_{1}, \ldots, e_{d}\right\}$ in such a way that $w_{k}=e_{1}+\cdots+e_{k}$ if $n(k)=0$, and $w_{k}=e_{1}+\cdots+e_{k-1}+\lambda_{n(k)}$ if $n(k)>0$. We denote the integers $n(k), m(k)$ and $p(k)$, defined in the Section 6 in terms of $\nu, k$ and $s$, simply by $n, m$, and $p$, respectively.

We begin by recalling some definitions and results from [4]. Let $\left\{m_{1}, \ldots, m_{d}\right\}$ be a basis of the lattice $M$. We consider a set of variables $\left\{c\left(m_{1}\right), \ldots, c\left(m_{d}\right)\right\} \cup\left\{u_{j}\left(m_{i}\right)\right\}_{i=1, \ldots, d}^{j \geqslant 1}$ to define the $\mathbf{C}$-algebra

$$
\mathcal{A}_{T_{N}}:=\mathbf{C}\left[c\left(m_{1}\right)^{ \pm 1}, \ldots, c\left(m_{d}\right)^{ \pm 1}\right] \otimes_{\mathbf{C}} \mathbf{C}\left[u_{j}\left(m_{i}\right)\right]_{i=1, \ldots, d}^{j \geqslant 1} .
$$

Since $\left\{m_{1}, \ldots, m_{d}\right\}$ form a basis of $M$ there is a unique homomorphism of semigroups

$$
M \rightarrow \mathcal{A}_{T_{N}}[[t]]^{*}
$$

given by $m_{i} \mapsto c\left(m_{i}\right) u\left(m_{i}\right)$ where $u\left(m_{i}\right)=1+\sum_{j \geqslant 1} u_{j}\left(m_{i}\right) t^{j}$ for $i=1, \ldots, d$. We associate to $w \in M$ the terms $c(w)$ and $u_{j}(w)$, for $j \geqslant 1$, in the ring $\mathcal{A}_{T_{N}}$, by $w \mapsto$ $c(w) u(w)$ in (7.1), where $c(w)$ is the constant term of the series and $u(w)$ is of the form $u(w)=1+\sum_{j \geqslant 1} u_{j}(w) t^{j}$. The following result show some relations among the elements $u_{i}(w) \in \mathcal{A}_{T_{N}}$, when we vary $i$ and $w \in M$, in terms of linear dependency relations among the $w \in M$.

Lemma 7·2. (see Lemma 6.2 in [4]). Let $w_{1}, \ldots, w_{k}$ be linearly independent vectors in the lattice $M$ spanning the linear subspace $\ell$ of $M_{\mathbf{Q}}$. For any $w \in \ell$ and $s \geqslant 1$ the term $u_{s}(w)$ belongs to $\mathbf{Q}\left[u_{1}\left(w_{j}\right), \ldots, u_{s}\left(w_{j}\right)\right]_{j=1}^{k}$.

We use in an essential manner the parametrization of the sets $H_{S, \nu}^{*}$ by the arc space of the torus $T_{N}$. We have an homomorphism of semigroups:

$$
\left(\sigma^{\vee} \cap M,+\right) \rightarrow\left(\mathcal{A}_{T_{N}}[[t]], \cdot\right) \text { given by } m \mapsto t^{\langle\nu, m\rangle} c(m) u(m) \text {, for } m \in \sigma^{\vee} \cap M .
$$

It defines a parametrization of $H_{\bar{S}, \nu}^{*}$ by the arc space of the torus $T_{N}$. Recall that by Lemma $3 \cdot 3$ we have that the analytic algebra of the germ $(S, 0)$ is $\mathcal{O}_{S}=\mathbf{C}\left\{\sigma^{\vee} \cap M_{0}\right\}[\zeta]$. The maximal ideal of $\mathcal{O}_{S}$ is $\left(x_{1}, \ldots, x_{d+1}\right)$ where $x_{i}:=X^{e_{i}}$ for $i=1, \ldots, d$ and $x_{d+1}:=\zeta$ (see the notations of Section (3). The restriction of the homomorphism $\mathbf{C}\left\{\sigma^{\vee} \cap M\right\} \rightarrow$ $\mathcal{A}_{T_{N}}[[t]]$ defined by (7.2) to the local algebra $\mathcal{O}_{S}$ parametrizes the set $H_{S, \nu}$. This homomorphism verifies that

$$
x_{i} \mapsto t^{\left\langle\nu, e_{i}\right\rangle} c\left(e_{i}\right) u\left(x_{i}\right),
$$


where $u\left(x_{i}\right)$ is a series of the form $u\left(x_{i}\right)=1+\sum_{j \geqslant 1} u_{j}\left(x_{i}\right)$. For $1 \leqslant i \leqslant d$ we have that $c\left(e_{i}\right) u\left(x_{i}\right)$ is the image of $e_{i}$ by the map (7.1), in particular $u\left(x_{i}\right)=u\left(e_{i}\right)$. We use the expansion (3.1) of $x_{d+1}=\sum \beta_{\lambda} X^{\lambda}$ as a power series in $\mathbf{C}\left\{\sigma^{\vee} \cap M\right\}$ to describe expansions of the terms $u_{r}\left(x_{d+1}\right)$ in terms of $u_{l}\left(e_{i}\right)$, for $i=1, \ldots, d+g$ and $1 \leqslant l \leqslant r$.

Notation $7 \cdot 3$. For $1 \leqslant j \leqslant g$ we denote by $C_{j}$ the $\mathbf{C}$-algebra of $\mathcal{A}_{T_{N}}$ generated by $c(m)$ for $m \in M_{j}$. We denote $C_{g}$ simply by $C$.

Notation 7.4. If $\nu \in \stackrel{\circ}{\sigma} \cap N$ and $r \geqslant 1$ are fixed we set the sequence $r_{1}, \ldots, r_{g}$ by:

$$
r_{1}=r, r_{2}=r_{1}-\left\langle\nu, \lambda_{2}-\lambda_{1}\right\rangle, \ldots, r_{g}=r_{1}-\left\langle\nu, \lambda_{g}-\lambda_{1}\right\rangle .
$$

Remark 7.5. Notice that if $r \leqslant s-\left\langle\nu, \lambda_{1}\right\rangle$ then we have that $r_{j} \leqslant s-\left\langle\nu, \lambda_{j}\right\rangle$ for every $1 \leqslant j \leqslant g$.

Proposition 7.6. Let $r \geqslant 1$ be an integer. In $\mathcal{A}_{T_{N}}$ we have the expansion:

$$
u_{r}\left(x_{d+1}\right)=u_{r}\left(\lambda_{1}\right)+\sum_{l+\left\langle\nu, \lambda-\lambda_{1}\right\rangle=r}^{\lambda \geqslant \lambda_{1}} \theta(\lambda) u_{l}(\lambda) \text {, with } \theta(\lambda):=\beta_{\lambda} \beta_{\lambda_{1}}^{-1} c(\lambda) c\left(\lambda_{1}\right)^{-1} .
$$

We use Notation $\%$. If $1 \leqslant j \leqslant g$ and $r_{j} \geqslant 0$ then we set

$$
\alpha_{r_{j}}^{j}:=\theta\left(\lambda_{j}\right) u_{r_{j}}\left(\lambda_{j}\right)+\sum_{\left\langle\nu, \lambda-\lambda_{j}\right\rangle+l_{j}=r_{j}}^{\left\langle\nu, \lambda_{j}\right\rangle<\langle\nu, \lambda\rangle \leqslant\left\langle\nu, \lambda_{j+1}\right\rangle, \lambda \neq \lambda_{j+1}} \theta(\lambda) u_{l_{j}}(\lambda),
$$

The following properties hold:

(i) $u_{r}\left(x_{d+1}\right)=\sum_{j=1, \ldots, g}^{r_{j} \geqslant 0} \alpha_{r_{j}}^{j}$.

(ii) The coefficient $\theta(\lambda)$ of a term $u_{l}(\lambda)$ in $\alpha_{r_{j}}^{j}$ belongs to $C_{j}$.

(iii) If the term $u_{l}(\lambda)$ appears in $\alpha_{r_{j}}^{j}$ then $1 \leqslant l<r_{j}$ unless $\lambda=\lambda_{j}$ and $l=r_{j}$, for $j=1, \ldots, g$.

Proof. We deduce that

$$
u\left(x_{d+1}\right)=\sum_{\lambda \geqslant \lambda_{1}} \beta_{\lambda} \beta_{\lambda_{1}}^{-1} c(\lambda) c\left(\lambda_{1}\right)^{-1} t^{\left\langle\nu, \lambda-\lambda_{1}\right\rangle}\left(\sum_{l \geqslant 0} u_{l}(\lambda) t^{l}\right) .
$$

by comparing the definition of $u\left(x_{d+1}\right)$ with the expansion (3.1) of $x_{d+1}$ in $\mathbf{C}\left\{\sigma^{\vee} \cap M\right\}$. The equality follows by collecting the terms in $t^{r}$ in (7.7). The sum in (7.5) is finite because there is a finite number of lattice points $\lambda$ verifying that $\lambda \geqslant \lambda_{1}$ and $\langle\nu, \lambda\rangle \leqslant\left\langle\nu, \lambda_{1}\right\rangle+r$, since $\nu \in \stackrel{\circ}{\sigma} \cap M$. Notice that $u_{0}(\lambda)=1$ by definition and the term obtained for $l=0$ (resp. $l=r$ ) in the sum (7.5) is $\sum_{\left\langle\nu, \lambda-\lambda_{1}\right\rangle=r} \beta_{\lambda} \beta_{\lambda_{1}}^{-1} c(\lambda) c\left(\lambda_{1}\right)^{-1}$ (resp. $u_{r}\left(\lambda_{1}\right)$ ). The other assertions are obtained by collecting the indices in the sum (7.5) according to (7.4).

Remark 7.7. For simplicity we convey that the term $\alpha_{r_{j}}^{j}$ equals zero whenever $r_{j}<0$. Hence we can write $u_{r}\left(x_{d+1}\right)=\sum_{j=1}^{g} \alpha_{r_{j}}^{j}$ for any $r \geqslant 1$

Proposition 7.8. (see Proposition 6.3 in [4]). If the vectors $w_{1}, \ldots, w_{k}$ in $M$ are linearly independent then the terms, $u_{i}\left(w_{1}\right), \ldots, u_{i}\left(w_{k}\right)$ for $i \geqslant 1$, are algebraically independent over the field of fractions of $C$, and in addition if $k=d$ they generate $\mathcal{A}_{T_{N}}$ as a $C$-algebra.

Proposition 7.9. Let us denote by $B_{0}$ the $\mathbf{C}$-algebra

$$
B_{0}:=\mathbf{C}\left[c\left(x_{i}\right), u_{1}\left(x_{i}\right), u_{2}\left(x_{i}\right), u_{3}\left(x_{i}\right), \ldots\right]_{i=1, \ldots d+1} .
$$


We set $F_{j}:=\left\{c(m) \mid m \in \sigma^{\vee} \cap M_{j}\right\}$ for $j=1, \ldots, g$. Suppose that for $1 \leqslant j \leqslant g$ the algebras $B_{0}, \ldots, B_{j-1}$ have been defined by induction. Then we have that $F_{j}$ is $a$ multiplicative subset of $B_{j-1}$ and the localization $B_{j}:=B_{j-1}\left[F_{j}^{-1}\right]$ is a subalgebra of $\mathcal{A}_{T_{N}}$ which is equal to $\mathcal{A}_{T_{N}}$ if $j=g$.

Proof. Notice that $\mathbf{C}\left[F_{j}\right]\left[F_{j}^{-1}\right]=C_{j}$. By definition we have that $c\left(x_{i}\right)=c\left(e_{i}\right)$ for $i=1, \ldots, d+1$ hence it follows that $F_{1} \subset B_{0}$ is a multiplicative subset. Then we apply Proposition [7.6 for $r=\left\langle\nu, \lambda_{2}-\lambda_{1}\right\rangle$. We deduce that $u_{r}\left(x_{d+1}\right)=\alpha_{r_{1}}+\theta\left(\lambda_{2}\right)$ where $\alpha_{r_{1}}$ belongs to $B_{1}$. It follows that $c\left(\lambda_{2}\right)=\beta_{\lambda_{1}} \beta_{\lambda_{2}}^{-1} c\left(\lambda_{1}\right) \theta\left(\lambda_{2}\right)$ belongs to $B_{1}$ and this implies that $F_{2}$ is a multiplicative subset of $B_{1}$. Suppose that the assertion is true for $1 \leqslant j-1 \leqslant g$. Then we apply Proposition $7 \cdot 6$ for $r=\left\langle\nu, \lambda_{j}\right\rangle$ and we deduce similarly that $F_{j}$ is a multiplicative subset of $B_{j-1}$. Finally, if $j=g$ we deduce that $B_{g}$ is a $C$-subalgebra of $\mathcal{A}_{T_{N}}$ (see Notation [7.3) which contains the set $\left\{u_{1}\left(e_{i}\right), u_{2}\left(e_{i}\right), u_{3}\left(e_{i}\right) \ldots\right\}_{i=1, \ldots, d}$. This set generate $\mathcal{A}_{T_{N}}$ as a $C$-algebra by Proposition $7 \cdot 8$, since the vectors $e_{1}, \ldots, e_{d}$ are linearly independent.

The idea of the proof of Theorem $5 \cdot 8$ is rather similar to that of Proposition $7 \cdot 9$, though we have to precise the form of the terms $u_{r}\left(x_{i}\right)$ which remains when taking a $s$-jet. This is controlled by the following Proposition:

Proposition 7·10. Suppose that $(\nu, s) \in A_{k}$. We consider an integer $r$ such that

$$
1 \leqslant r \leqslant \begin{cases}s-\left\langle\nu, \lambda_{1}\right\rangle & \text { if } n=0 \\ s-\left\langle\nu, e_{m}-\lambda_{n}+\lambda_{1}\right\rangle & \text { if } n>0 \text { and } m \neq 0 \\ s-\left\langle\nu, \lambda_{n}-\lambda_{1}\right\rangle & \text { if } n>0 \text { and } m=0\end{cases}
$$

The terms $\alpha_{j}^{r_{j}}$ in the expansion $u_{r}\left(x_{d+1}\right)=\sum_{j=1}^{g} \alpha_{j}^{r_{j}}$ vanish for $p<j \leqslant g$. If in addition, the term $u_{l}(\lambda)$ appears with non zero coefficient in the expansion of $\alpha_{i}^{r_{i}}$, for some $1 \leqslant i \leqslant p$, then

$$
\lambda \in \ell_{\nu}^{s} \cap M_{i} \text { and } l \leqslant s-\left\langle\nu, e_{q_{\lambda}}\right\rangle .
$$

Proof. By Proposition $7 \cdot 6$ we have that a term $\alpha_{j}^{r_{j}}$ may be non-zero in the expansion of $u_{r}\left(x_{d+1}\right)$ if $r_{j} \geqslant 0$ (see Notation 7.4). By Remark 7.5 this happens if

$$
0 \leqslant \begin{cases}s-\left\langle\nu, \lambda_{j}\right\rangle & \text { if } n=0 \\ s-\left\langle\nu, e_{m}-\lambda_{n}+\lambda_{j}\right\rangle & \text { if } n>0 \text { and } m \neq 0 \\ s-\left\langle\nu, \lambda_{n}-\lambda_{j}\right\rangle & \text { if } n>0 \text { and } m=0\end{cases}
$$

This holds if and only if $j \leqslant p$ (see Notation 7.1).

Suppose that $u_{l}(\lambda)$ appears with non zero coefficient in $\alpha_{j}^{r_{j}}$ for some $1 \leqslant j \leqslant p$. This implies that $X^{\lambda}$ appears in the expansion (3.1) of $\zeta,\left\langle\nu, \lambda_{j}\right\rangle \leqslant\langle\nu, \lambda\rangle \leqslant\left\langle\nu, \lambda_{j+1}\right\rangle$ and $\lambda \neq \lambda_{j+1}$, thus $\lambda \in M_{j}$ and $\lambda \leqslant{ }_{\nu} \lambda_{j}$. Then we obtain

$$
0 \leqslant r_{j}=\left\langle\nu, \lambda-\lambda_{j}\right\rangle+l \leqslant \begin{cases}s-\left\langle\nu, \lambda_{j}\right\rangle & \text { if } n=0 \\ s-\left\langle\nu, e_{m}-\lambda_{n}+\lambda_{j}\right\rangle & \text { if } n>0 \text { and } m \neq 0 \\ s-\left\langle\nu, \lambda_{n}-\lambda_{j}\right\rangle & \text { if } n>0 \text { and } m=0\end{cases}
$$

We deduce from these inequalities, using (6.5), $\lambda_{j} \leqslant \nu$ and $\lambda_{n} \leqslant{ }_{\nu} \lambda_{p}$ that

$$
l \leqslant s-\left\langle\nu, e_{q_{\lambda_{j}}}\right\rangle .
$$

Since $\lambda \in M_{j}$ is $\geqslant_{\nu} \lambda_{j}$ we have a unique expansion of the form $\lambda=\lambda^{\prime}+\sum_{e_{i} \notin \ell\left(\lambda_{j}\right)}^{1 \leqslant i \leqslant d} h_{i} e_{i}$, 
where $\lambda^{\prime} \geqslant \lambda_{j}$ belongs to $\ell\left(\lambda_{j}\right)$ and $h_{i} \geqslant 0$ are integers. Since $\langle\nu, \lambda\rangle \leqslant s$ it follows from Proposition 6.10 that $\lambda$ belongs to $\ell_{\nu}^{s}$. We deduce that $\langle\nu, \lambda\rangle \geqslant\left\langle\nu, \lambda^{\prime}\right\rangle$ and $\langle\nu, \lambda\rangle \geqslant\left\langle\nu, e_{i}\right\rangle$ if $h_{i}>0$. This implies that $l \leqslant s-\left\langle\nu, e_{i}\right\rangle$ if $h_{i} \neq 0$ and together with (7.9) proves that $l \leqslant s-\left\langle\nu, e_{q_{\lambda}}\right\rangle$.

Notation $7 \cdot 11$. We denote by $\bar{B}_{0}$ the $\mathbf{C}$-algebra of $\mathcal{A}_{T_{N}}$ generated by:

$$
c\left(x_{i}\right), u_{1}\left(x_{i}\right), \ldots, u_{s-\left\langle\nu, e_{i}\right\rangle}\left(x_{i}\right) \text { for those } 1 \leqslant i \leqslant d+1 \text { such that }\left\langle\nu, e_{i}\right\rangle \leqslant s .
$$

We set $\bar{C}$ the $\mathbf{C}$-algebra generated by $\left\{c\left(e_{j}\right) \mid\left\langle\nu, e_{j}\right\rangle \leqslant s, 1 \leqslant j \leqslant d+p\right\}$

Proposition 7.12. We use Notations $7 \cdot 1$ and $\% \cdot 11$. The $\bar{C}$-algebra generated by $\bar{B}_{0}$ coincides with the $\bar{C}$-algebra of $\mathcal{A}_{T_{N}}$ generated by the following elements:

$$
\left\{u_{1}\left(e_{i}\right), \ldots, u_{s-\left\langle\nu, e_{i}\right\rangle}\left(e_{i}\right)\right\}_{i=1, \ldots, k-1}
$$

together with

$$
\begin{cases}\left\{u_{l}\left(e_{k}\right)\right\}_{l=1}^{s-\left\langle\nu, e_{k}\right\rangle} & \text { if } n=0 \\ \left\{u_{l}\left(e_{m}\right)\right\}_{l=1}^{s-\left\langle\nu, e_{i}\right\rangle} \cup\left\{u_{l}\left(x_{d+1}\right)\right\}_{l=s-\left\langle\nu, e_{m}-\lambda_{n}+\lambda_{1}\right\rangle+1}^{s-\left\langle\nu, \lambda_{1}\right\rangle} & \text { if } n>1 \text { and } m \neq 0, \\ \left\{u_{l}\left(x_{d+1}\right)\right\}_{l=\left\langle\nu, \lambda_{n}\right\rangle+1}^{s-\left\langle\nu, \lambda_{1}\right\rangle} & \text { if } n>0 \text { and } m=0 .\end{cases}
$$

Proof. We consider the case $n=0$ (resp. $n>0$ and $m=0$ ). By Lemma 6.10 we have that if $\left\langle\nu, e_{i}\right\rangle \leqslant s$ and $1 \leqslant i \leqslant d$ then $1 \leqslant i \leqslant k$. (resp. $1 \leqslant i \leqslant k-1$ ). It is sufficient to prove that the elements $u_{r}\left(x_{d+1}\right)$ for $1 \leqslant r \leqslant s-\left\langle\nu, \lambda_{1}\right\rangle$ belong to the $\bar{C}$-algebra generated by (7.11) and (7.12). By Propositions $7 \cdot 6$ and 7.10 we get $u_{r}\left(x_{d+1}\right)=\sum \theta(\lambda) u_{l}(\lambda)$ where $\lambda \in \ell_{\nu}^{s} \cap M_{p}, \theta(\lambda) \in \bar{C}$ and $l \leqslant s-\left\langle\nu, e_{q_{\lambda}}\right\rangle$. This implies that $\lambda \in \operatorname{span}_{\mathbf{Q}}\left(e_{1}, \ldots, e_{k}\right)$ (resp. $\left.\lambda \in \operatorname{span}_{\mathbf{Q}}\left(e_{1}, \ldots, e_{k-1}\right)\right)$ and $l \leqslant s-\left\langle\nu, e_{i}\right\rangle$ for $i=1, \ldots, k$ (resp. for $i=1, \ldots, k-1$ ) thus by Lemma $7 \cdot 2$ we deduce that $\theta(\lambda) u_{l}(\lambda) \in \bar{C}\left[u_{1}\left(e_{i}\right), \ldots, u_{s-\left\langle\nu, e_{i}\right\rangle}\left(e_{i}\right)\right]_{i=1 \ldots k(\text { resp. } k-1)}$.

We deal then with the case $n>0$ and $m \neq 0$. It is sufficient to prove that the elements $u_{r}\left(x_{d+1}\right)$ for $1 \leqslant r \leqslant s-\left\langle\nu, e_{m}-\lambda_{n}+\lambda_{1}\right\rangle$ belong to the $\bar{C}$-algebra generated by (7.11) and (17.12). The conclusion follows by the same arguments, by using Propositions [7.6. $7 \cdot 10$ and Lemma $7 \cdot 2$,

Proposition 7·13. We set $\bar{F}_{j}:=\left\{c\left(e_{i}\right) \mid\left\langle\nu, e_{i}\right\rangle \leqslant s, 1 \leqslant i \leqslant d+j\right\}$ for $j=1, \ldots, p$. Suppose that for some $1 \leqslant j<p$ the algebras $\bar{B}_{0}, \ldots, \bar{B}_{j-1}$ have been defined by induction. Then $\bar{F}_{j}$ is a multiplicative subset of $\bar{B}_{j-1}$ and the localization $\bar{B}_{j}:=\bar{B}_{j-1}\left[\left(\bar{F}_{j}\right)^{-1}\right]$ is a subalgebra of $\mathcal{A}_{T_{N}}$. Then the ring $\bar{B}:=\bar{B}_{p}$ is generated by $\bar{B}_{0}$ as a $\bar{C}$-algebra. The ring $\bar{B}$ is the coordinate ring of $j_{s}\left(H_{\nu}^{*}\right)$.

Proof. Set $\bar{C}_{j}:=\mathbf{C}\left[\bar{F}_{j}\right]\left[\left(\bar{F}_{j}\right)^{-1}\right]$. By definition we have that $c\left(x_{i}\right)=c\left(e_{i}\right)$ for $i=1, \ldots, d+$ 1, hence it follows that $\bar{F}_{1} \subset \bar{B}_{0}$ is a multiplicative subset. We apply Propositions [7.6] and $7 \cdot 10$ for $r=\left\langle\nu, \lambda_{2}-\lambda_{1}\right\rangle$. We deduce that $u_{r}\left(x_{d+1}\right)=\alpha_{r_{1}}+\theta\left(\lambda_{2}\right)$ where $\alpha_{r_{1}}$ belongs to $\bar{B}_{1}$. It follows that $c\left(\lambda_{2}\right)=\beta_{\lambda_{1}} \beta_{\lambda_{2}}^{-1} c\left(\lambda_{1}\right) \theta\left(\lambda_{2}\right)$ belongs to $\bar{B}_{1}$ and this implies that $\bar{F}_{2}$ is a multiplicative subset of $\bar{B}_{1}$. Suppose that the assertion is true for $1 \leqslant j-1 \leqslant g$. Then we apply Proposition 7.10 for $r=\left\langle\nu, \lambda_{j}\right\rangle$ and we deduce similarly that $\bar{F}_{j}$ is a multiplicative subset of $\bar{B}_{j-1}$. We can iterate this procedure for $j=1, \ldots, p$. By Proposition $7 \cdot 12$ it follows that $\bar{B}$ is the $\bar{C}$-algebra generated by $\bar{B}_{0}$. By construction $\bar{B}$ is the coordinate ring of $j_{s}\left(H_{\nu}^{*}\right)$ (compare with Proposition (7.9).

Lemma 7·14. The generators (7.11) and (7.12) of the $\bar{C}$-algebra $\bar{B}$ are algebraically independent over the field of fractions of $\bar{C}$. 
Proof. If $n>0$ and if $\left\langle\nu, \lambda_{n}\right\rangle<r$ then we have that $u_{r}\left(x_{d+1}\right)$ is of the form:

$$
u_{r}\left(x_{d+1}\right)=\theta_{\lambda_{n}} u_{r_{n}}\left(\lambda_{n}\right)+\sum_{\lambda \neq \lambda_{n}}^{l \leqslant r_{n}} \theta_{\lambda} u_{l}(\lambda)+\sum_{e_{1} \wedge \cdots \wedge e_{k-1} \wedge \lambda=0} \theta_{\lambda} u_{r_{n}}(\lambda) .
$$

(see Notation 7.4). By Proposition 7.6 we have that $u_{r}\left(x_{d+1}\right)=\sum_{j=1}^{g} \alpha_{r_{j}}^{j}$ and if $u_{l}(\lambda)$ appears in the expansion (7.6) of $\alpha_{r_{j}}^{j}$ with non zero coefficient then $l=r-\left\langle\nu, \lambda-\lambda_{1}\right\rangle$ and $\lambda \in M_{j}$ verifies that

$$
\left\langle\nu, \lambda_{j}\right\rangle<\langle\nu, \lambda\rangle \leqslant\left\langle\nu, \lambda_{j+1}\right\rangle .
$$

If $1 \leqslant j<n$ then we have the inequality $l=r-\left\langle\nu, \lambda-\lambda_{1}\right\rangle \leqslant r-\left\langle\nu, \lambda_{n}-\lambda_{1}\right\rangle=r_{n}$. The equality $l=r_{n}$ implies that $\langle\nu, \lambda\rangle=\left\langle\nu, \lambda_{n}\right\rangle$, thus $\lambda$ belongs to $M_{n-1} \cap \operatorname{span}_{\mathbf{Q}}\left(e_{1}, \ldots, e_{k-1}\right)$ since $\left\langle\nu, \lambda_{n}\right\rangle<\left\langle\nu, e_{m}\right\rangle$. If $n \leqslant j$ then we have that $l<r_{n}$ by (7.14).

Now we prove the Proposition by distinguishing the following cases:

(i) If $n=0$ then the assertion is consequence of Proposition $7 \cdot 8$

(ii) If $n>0$ and $m=0$ the assertion is consequence of (7.13) and Proposition $7 \cdot 8$

(iii) If $n>0$ and $m \neq 0$ then by Proposition 7.2 and the definition of $e_{m}$ we have that the $\bar{C}$ algebra generated by $\left\{u_{l}\left(e_{1}\right), \ldots, u_{l}\left(e_{k-1}\right)\right\}_{l=1}^{s-\left\langle\nu, e_{i}\right\rangle} \cup\left\{u_{l}\left(e_{m}\right)\right\}_{l=1}^{s-\left\langle\nu, e_{m}\right\rangle}$, coincides with the $\bar{C}$ algebra generated by $\left\{u_{l}\left(e_{i}\right)\right\}_{l=1, \ldots, s-\left\langle\nu, e_{i}\right\rangle}^{i=1, \ldots, k-1} \cup\left\{u_{l}\left(\lambda_{n}\right)\right\}_{l=1}^{s-\left\langle\nu, e_{m}\right\rangle}$. We deduce the statement from this, the equation (17.13) and Proposition $7 \cdot 8$

Proof of Theorem 5.8. The statement is clear if $(\nu, s) \in A_{0}$. Suppose that $(\nu, s) \in A_{k}$ for some $1 \leqslant k \leqslant d$ and $s>0$. By Lemma $7 \cdot 14$ we have $s k-$ ord $\mathcal{J}_{k}(\nu)$ generators of $\bar{B}$ as a $\bar{C}$ algebra. These generators, indicated in (17.11) and (7.12), are algebraically independent over the field of fractions of $\bar{C}$. Notice that $\bar{C}$ is the $\mathbf{C}$ algebra of the rank $k$ sublattice of $\ell_{\nu}^{s} \cap M_{p}$ hence Spec $\bar{C}$ is a $k$-dimensional torus. It follows from this and Proposition $7 \cdot 13$ that we can define an isomorphic parametrization

$$
\left(\mathbf{C}^{*}\right)^{k} \times \mathbf{A}_{\mathbf{C}}^{s k-\operatorname{ord}_{\mathcal{J}_{k}}(\nu)} \rightarrow \operatorname{Spec} \bar{B} \cong j^{s}\left(H_{\nu}^{*}\right) .
$$

\section{Description of the series $P(S)$ and proofs of the rationality results}

First we study the relations between the sets $j_{s}\left(H_{\nu}^{*}\right)$ when $\nu$ varies.

Definition 8.1. Define an equivalence relation in the set $A_{k}$ for $1 \leqslant k \leqslant d$ :

$$
(\nu, s) \sim\left(\nu^{\prime}, s\right) \in A_{k} \Leftrightarrow\left\{\begin{array}{c}
s=s^{\prime}, \nu \text { and } \nu^{\prime} \text { define the same face of } \mathcal{N}\left(\mathcal{J}_{j}\right) \\
\text { and } \operatorname{ord}_{\mathcal{J}_{j}}(\nu)=\operatorname{ord}_{\mathcal{J}_{j}}\left(\nu^{\prime}\right) \text { for } 1 \leqslant j \leqslant k .
\end{array}\right.
$$

We denote by $[(\nu, s)]$ the equivalence class of $(\nu, s) \in A_{k}$ by this relation.

Remark $8 \cdot 2$.

(i) The set $\left\{\left[\left(\nu, s_{0}\right)\right] \mid\left(\nu, s_{0}\right) \in A_{k}\right\}$ is finite for $s_{0}>0$ and $1 \leqslant k \leqslant d$.

(ii) If $k=d$ the equivalence relation is the equality.

Proposition 8.3. (cf. Prop. 8.3 [4]) Let $(\nu, s),\left(\nu^{\prime}, s\right) \in A_{k}$. The following are equivalent:

(i) $(\nu, s) \sim\left(\nu^{\prime}, s\right)$.

(ii) $\ell_{\nu}^{s}=\ell_{\nu^{\prime}}^{s}$ and $\nu_{\mid \ell_{\nu}^{s}}=\nu_{\mid \ell_{\nu^{\prime}}^{s}}^{\prime}$. 
(iii) $j_{s}\left(H_{\nu}^{*}\right)=j_{s}\left(H_{\nu^{\prime}}^{*}\right)$.

(iv) $j_{s}\left(H_{\nu}^{*}\right) \cap j_{s}\left(H_{\nu^{\prime}}^{*}\right) \neq \emptyset$.

Proof. The equivalence between (i) and (ii) follows by Lemma 6.10, Definition 5.7 and the definition of the equivalence relation.

If (ii) holds, then the vectors obtained in the algorithm in Proposition 6.5 coincides for both $\nu$ and $\nu^{\prime}$. It follows that the indices $m(k), n(k)$ and $p(k)$ are the same for $\nu$ and $\nu^{\prime}$. These facts imply that the isomorphic parametrizations (7.15) corresponding to $(\nu, s),\left(\nu^{\prime}, s\right) \in A_{k}$ coincide. Hence $j_{s}\left(H_{\nu}^{*}\right)=j_{s}\left(H_{\nu^{\prime}}^{*}\right)$ and (iii) holds.

If (iv) holds we prove that (ii) holds. There exists $h \in H_{\nu}^{*}$ and $h^{\prime} \in H_{\nu^{\prime}}^{*}$ such that $j_{s}(h)=j_{s}\left(h^{\prime}\right)$. Then for any $i \in\{1, \ldots, d+1\}$ the inequality $\left\langle\nu, e_{i}\right\rangle \leqslant s$ implies that $\left\langle\nu, e_{i}\right\rangle=\left\langle\nu^{\prime}, e_{i}\right\rangle$. We denote by $n(k)$ and $m(k)$ (resp. $n^{\prime}(k)$ and $m^{\prime}(k)$ ) the integers associated to $(\nu, s)$ (resp. $\left.\left(\nu^{\prime}, s\right)\right)$ in Proposition 6.5. If $n(k)=0$ then the assertion follows. If $n(k)>0$ we set $\ell=\operatorname{span}_{\mathbf{Q}}\left\{e_{i} \mid 1 \leqslant i \leqslant d,\left\langle\nu, e_{i}\right\rangle \leqslant s\right\}$ and $x_{d+1}^{\prime}=\sum_{\lambda_{n(k)} \nless \lambda, \lambda \in \ell} \beta_{\lambda} X^{\lambda}$. We have that $\nu_{\mid \ell}=\nu_{\mid \ell}^{\prime}$. If $\beta_{\lambda} \neq 0$ appears in $x_{d+1}^{\prime}$ and $\lambda=\sum_{i=1}^{d} a_{i} e_{i}$ then $\langle\nu, \lambda\rangle \geqslant\left\langle\nu, e_{i}\right\rangle$ whenever $a_{i} \neq 0$ (this is consequence of Lemma 6.10). In addition, if $\langle\nu, \lambda\rangle \leqslant s$ then it follows by Lemma $\mathbf{7 \cdot 2}$ that $u_{r}(\lambda) \in \mathbf{Q}\left[u_{1}\left(e_{i}\right), \ldots, u_{s-\left\langle\nu, e_{i}\right\rangle}\left(e_{i}\right)\right]_{1 \leqslant i \leqslant d}^{\left\langle\nu, e_{i}\right\rangle \leqslant s}$ for $r=1, \ldots, s-$ $\langle\nu, \lambda\rangle$. Then it is easy to see that the initial coefficients of $X^{\lambda_{j}} \circ h$ and $X^{\lambda_{j}} \circ h^{\prime}$ coincide for $i=1, \ldots, n(k)-1$. We deduce that $j_{s}\left(x_{d+1}^{\prime} \circ h\right)=j_{s}\left(x_{d+1}^{\prime} \circ h^{\prime}\right)$ hence $j_{s}\left(\left(x_{d+1}-x_{d+1}^{\prime}\right) \circ h\right)$ is of order $\left\langle\nu, \lambda_{n(k)}\right\rangle$. Since $j_{s}\left(\left(x_{d+1}-x_{d+1}^{\prime}\right) \circ h\right)=j_{s}\left(\left(x_{d+1}-x_{d+1}^{\prime}\right) \circ h^{\prime}\right)$ we deduce that $\left\langle\nu, \lambda_{n(k)}\right\rangle=\left\langle\nu^{\prime}, \lambda_{n(k)}\right\rangle$ hence $\lambda_{n(k)}=\lambda_{n^{\prime}(k)}$ and (ii) holds.

Notation 8.4. The cone $\hat{\sigma}:=\sigma \times \mathbf{R}_{\geqslant 0}$ is rational for the lattice $\hat{N}:=N \times \mathbf{Z}$.

(i) If $\tau \subset \sigma$ and $1 \leqslant k \leqslant d$ we set

$$
\tau(k):=\left\{(\nu, s) \mid \nu \in \stackrel{\circ}{\tau} \cap \stackrel{\circ}{\sigma} \text { and } \phi_{k}(\nu) \leqslant s<\phi_{k+1}(\nu)\right\} .
$$

(ii) If $\tau \in \cap_{i=1}^{k} \Sigma_{i}$ then we set $A_{k, \tau}:=\tau(k) \cap \hat{N}$.

Remark 8.5. If $\tau$ is a cone contained in a cone of the fan $\cap_{i=1}^{k} \Sigma_{i}$ then we have that if $\tau(k) \neq \emptyset$, then the closure of $\tau(k)$ in $\hat{\sigma}$ is a convex polyhedral cone, rational for the lattice $\hat{N}$ (since in this case the functions ord $\mathcal{J}_{1}, \ldots$ ord $\mathcal{J}_{k}$, hence also $\phi_{1}, \ldots, \phi_{k}$, are linear on $\tau$ and the function ord $\mathcal{J}_{k+1}$, hence also $\phi_{k+1}$, is piece-wise linear and convex on $\tau)$. The set $A_{k, \tau}$ may be empty, for instance, if $\tau$ is contained in the boundary of $\sigma$ or if for all $\nu$ in the interior of $\tau$ we have that $\phi_{k}(\nu)=\phi_{k+1}(\nu)$.

\section{Remark 8.6.}

(i) For $1 \leqslant k \leqslant d$ we have that $A_{k}=\bigsqcup_{\tau \in \cap_{i=1}^{k} \Sigma_{i}} A_{k, \tau}$.

(ii) The vectors $(\nu, s),\left(\nu^{\prime}, s\right) \in A_{k}$ are equivalent by relation $\sim$ in (8.1) if and only if there exists a cone $\tau \in \cap_{i=1}^{k} \Sigma_{i}$ such that $\nu$ and $\nu^{\prime}$ belong to the relative interior of $\tau$ and we have that $\phi_{i}(\nu)=\phi_{i}\left(\nu^{\prime}\right)$, for $i=1, \ldots, k$.

(iii) It follows that $A_{k} / \sim=\bigsqcup_{\tau \in \cap_{i=1}^{k} \Sigma_{i}} A_{k, \tau} / \sim$, where $A_{k, \tau} / \sim$ is the set of equivalent classes of elements in the set $A_{k, \tau}$ by relation (8.1).

Proposition 8.7. (cf. Prop. 8.7 [4]) If $\nu \in \stackrel{\circ}{\sigma}, s \geqslant 1$ and $\theta \leqslant \sigma$ then the following relations are equivalent:

(i) $j_{s}\left(H_{\nu}^{*}\right) \cap j_{s}\left(H_{S_{\theta}}\right) \neq \emptyset$,

(ii) $j_{s}\left(H_{\nu}^{*}\right) \subset j_{s}\left(H_{S_{\theta}}\right)$,

(iii) $\ell_{\nu}^{s} \subset \theta^{\perp}$. 
Proof. If (i) holds, there is an arc $h \in H_{\nu}^{*}$ with $j_{s}(h) \in j_{s}\left(H_{S_{\theta}}\right)$. Then if $j_{s}\left(X^{e_{i}} \circ h\right) \neq 0$ the vector $e_{i}$ belongs to $\theta^{\perp}$. By Lemma $6 \cdot 10$ those vectors generate the subspace $\ell_{\nu}^{s}$ and hence (iii) holds.

Suppose that (iii) holds. The vector $\nu^{\prime}:=\nu_{\mid \theta^{\perp}}$ belongs to the lattice $N(\theta, \zeta)$ and it is in the interior of the cone $\sigma / \theta \mathbf{R}$ (see Notation 3.4). By definition we have that $\ell_{\nu}^{s}=\ell_{\nu^{\prime}}^{s}$ and $\nu_{\mid \ell_{\nu}^{s}}=\nu_{\mid \ell_{\nu^{\prime}}^{s}}^{\prime}$. Then we continue as in the proof of (iv) $\Rightarrow$ (ii) in Proposition $8 \cdot 3$.

Proposition 8.8. If $1 \leqslant k \leqslant d$ and $(\nu, s) \in A_{k}$ then the following assertions are equivalent:

(i) The intersection $j_{s}\left(H_{\nu}^{*}\right) \cap\left(\bigcup_{0 \neq \theta \leqslant \sigma} j_{s}\left(H_{S_{\theta}}\right)\right)$ is empty.

(ii) The face $\mathcal{F}_{\nu}$ of the polyhedron $\mathcal{N}\left(\mathcal{J}_{k}\right)$ determined by $\nu$ is contained in the interior of $\sigma^{\vee}$.

Proof. The proof coincides with that of Proposition 8.8 [4].

Definition 8.9. (cf. Def. 8.9 [4]) If $1 \leqslant k \leqslant d$ we define the set $\mathcal{D}_{k}$ as the subset of cones $\tau \in \bigcap_{i=1}^{k} \Sigma_{i}$ such that the face $\mathcal{F}_{\tau}$ of $\mathcal{N}\left(\mathcal{J}_{k}\right)$ is contained in the interior of $\sigma^{\vee}$.

Remark 8.10. Notice that $\mathcal{D}_{d}=\bigcap_{i=1}^{d} \Sigma_{i}$. If $\tau \in \mathcal{D}_{d}$, the set $\tau(d)$ is non-empty if and only if $\stackrel{\circ}{\tau} \subset \stackrel{\circ}{\sigma}$.

As a consequence of the results of this Section we have the following Propositions:

Proposition 8-11. (cf. Prop. 8.11 [4]) Let us fix an integer $s_{0} \geqslant 1$. We have the following partition as union of locally closed subsets:

$$
j_{s_{0}}\left(H_{S}^{*}\right) \backslash \bigcup_{0 \neq \theta \leqslant \sigma} j_{s_{0}}\left(H_{S_{\theta}}\right)=\bigsqcup_{k=1}^{d} \bigsqcup_{\tau \in \mathcal{D}_{k}\left[\left(\nu, s_{0}\right)\right] \in A_{k, \tau} / \sim} j_{s_{0}}\left(H_{\nu}^{*}\right)
$$

If $s_{0} \geqslant 1$ the coefficient of $T^{s_{0}}$ in the auxiliary series $P(S)$ is obtained by taking classes in the Grothendieck ring in (8.2), and then using Theorem $5 \cdot 8$

Proposition 8·12. (cf. Prop. 8.12 [4]) If $\tau \in \mathcal{D}_{k}$ we set

$$
P_{k, \tau}(S)=(\mathbf{L}-1)^{k} \sum_{s \geqslant 1} \sum_{\left[\left(\nu, s_{0}\right)\right] \in A_{k, \tau} / \sim} \mathbf{L}^{s k-\text { ord } \mathcal{J}_{k}(\nu)} T^{s} .
$$

We have that $P(S)=\sum_{k=1}^{d} \sum_{\tau \in \mathcal{D}_{k}} P_{k, \tau}(S)$.

The main results on the geometric motivic Poincaré series are based on Theorem $5 \cdot 8$ and Proposition 8.11. The proofs of the main results of this Section follow by applying the method introduced in the toric case [4].

Recall that if $\rho \in \cap \Sigma_{j}$ is of dimension one we denote by $\nu_{\rho}$ the integral primitive vector in $N$.

Proposition 8.13. (cf. Prop. 9.5 [4]) If $1 \leqslant k \leqslant d-1$ then the rational form of the series $P_{k, \tau}(S)$ is of the form:

$$
\frac{Q_{S, k, \tau}}{\prod_{\rho \leqslant \tau}^{\operatorname{dim} \rho=1}\left(1-\mathbf{L}^{\Psi_{k}\left(\nu_{\rho}\right)} T^{\phi_{k}\left(\nu_{\rho}\right)}\right) \prod_{\rho \in \Sigma_{k+1}, \rho \subset \tau,}^{\operatorname{dim} \rho=1, \phi_{k+1}\left(\nu_{\rho}\right) \neq \phi_{k}\left(\nu_{\rho}\right)}\left(1-\mathbf{L}^{\Psi_{k+1}\left(\nu_{\rho}\right)} T^{\phi_{k+1}\left(\nu_{\rho}\right)}\right)},
$$

for some $Q_{S, k, \tau} \in \mathbf{Z}[\mathbf{L}, T]$. If $k=d$ then equation (8.3) holds by replacing in the denominator the term $\prod_{\rho \in \Sigma_{k+1}, \rho \subset \tau}^{\operatorname{dim} \rho=1}\left(1-\mathbf{L}^{\Psi_{k+1}\left(\nu_{\rho}\right)} T^{\phi_{k+1}\left(\nu_{\rho}\right)}\right)$ by $1-\mathbf{L}^{d} T$. Both numerator and 
denominator of (8.3) are determined by the lattice $M$ and the Newton polyhedra of the logarithmic jacobian ideals.

Proof. The proof follows by using the same argument of Proposition 9.5 [4].

Remark 8.14. The factor $1-\mathbf{L}^{d} T$ does not appear in the denominator of $P_{k, \tau}(S)$ for $1 \leqslant k \leqslant d-1$.

Proof of Theorem 5.9. It is consequence of Propositions 8.13 and 8.12

Proof of Corollary 5.10. It follows from Proposition 5.4 and Theorem 5.9.

Proof of Corollary [5.11] The proof is the same as Proposition 10.1 [4].

\section{Geometrical definition of the logarithmic jacobian ideals}

We introduce a distinguished sequence of monomial ideals of the coordinate ring of the normalization of the q.o. hypersurface.

Let $(Y, 0)$ be a germ of complex analytic variety. Its analytic algebra $\mathcal{O}_{Y}$ is of the form $\mathcal{O}_{Y}=\mathbf{C}\{X\} / I$, for $X=\left(X_{1}, \ldots, X_{n}\right)$. We denote by $\Omega_{Y}^{1}$ the $A$-module of (Kähler) holomorphic differential forms and by $d: \mathcal{O}_{Y} \rightarrow \Omega_{Y}^{1}$ its canonical derivation. We denote by $\Omega_{Y}^{k}$ the $A$-module $\Omega_{Y}^{k}:=\bigwedge^{k} \Omega_{Y}^{1}$. See Section 1.10 of [21] for instance.

First, we review the normal toric case following [36] Chapter 3 and [28 Appendix. We consider the toric singularity $Z$ with analytic algebra of the form $\mathcal{O}_{Z}=\mathbf{C}\left\{\sigma^{\vee} \cap M\right\}$. We denote by $D$ the equivariant Weil divisor defined by the sum of orbit closures of codimension one in the toric variety $Z$. The $\mathcal{O}_{Z}$-module $\Omega_{Z}^{1}(\log D)$ of 1 -forms of $Z$ with logarithmic poles along $D$ is identified with $\mathcal{O}_{Z} \otimes_{\mathbf{Z}} M$. We have a map of $\mathcal{O}_{Z}$-modules $\omega: \Omega_{Z}^{1} \rightarrow \mathcal{O}_{Z} \otimes_{\mathbf{Z}} M$, determined by $d X^{\gamma} \mapsto X^{\gamma} \otimes \gamma$, for $\gamma \in \sigma^{\vee} \cap M$. Notice that if $\left\{\gamma_{i}\right\}_{i=1}^{r}$ generate the semigroup $\sigma^{\vee} \cap M$ then $\left\{d X^{\gamma_{i}}\right\}_{i=1}^{r}$ generate the $\mathcal{O}_{Z}$-module $\Omega_{Z}^{1}$. If $\psi=\sum_{\gamma \in \sigma^{\vee} \cap M} c_{\gamma} X^{\gamma}$ then $d \psi \mapsto \omega(d \psi)=\sum_{\gamma \in \sigma^{\vee} \cap M} c_{\gamma} X^{\gamma} \otimes \gamma$. Notice that $\omega(d \psi)=\sum_{i=1}^{d}\left(\sum_{\gamma \in \sigma^{\vee} \cap M} c_{\gamma} \gamma_{i} X^{\gamma}\right) \otimes u_{i}$, where $\left(\gamma_{1}, \ldots, \gamma_{d}\right)$ denote the coordinates of $\gamma$ in terms of a basis $u_{1}, \ldots, u_{d}$ of the lattice $M$. For $k=1, \ldots, d$ we have the following homomorphism of $\mathcal{O}_{Z}$-modules $\wedge^{k} \omega: \Omega_{Z}^{k} \longrightarrow \Omega_{Z}^{k}(\log D)=\mathcal{O}_{Z} \otimes \mathbf{z} \wedge^{k} M$

$$
d X^{\gamma_{1}} \wedge \cdots \wedge d X^{\gamma_{k}} \mapsto X^{\gamma_{1}+\cdots+\gamma_{k}} \otimes \gamma_{1} \wedge \cdots \wedge \gamma_{k} .
$$

Notice that fixing a basis $u_{1}, \ldots, u_{d}$ of the rank $d$ lattice $M$ defines an isomorphism $\bigwedge^{d} M \rightarrow \mathbf{Z}$, given by $u_{1} \wedge \cdots \wedge u_{d} \mapsto 1$. This provides an homomorphism of $\mathcal{O}_{Z}$-modules

$$
\phi: \Omega_{Z}^{d}(\log D) \rightarrow \mathcal{O}_{Z} \text {. }
$$

The image of $\Omega_{Z}^{d}$ by the composite $\Omega_{Z}^{d} \rightarrow \Omega_{Z}^{d}(\log D) \rightarrow \mathcal{O}_{Z}$ is an ideal of $\mathcal{O}_{Z}$, which is independent of the basis of $M$ chosen. This ideal is called the logarithmic jacobian ideal of $Z$ in $[\mathbf{2 8}$.

If $(S, 0)$ is the germ of q.o. singularity then its normalization is a toric singularity and we have a canonical map $\eta: \Omega_{S}^{k} \rightarrow \Omega_{\bar{S}}^{k}$ for $1 \leqslant k \leqslant d$, which induced by the normalization map $\bar{S} \rightarrow S$. We denote also by $\phi$ and $\wedge^{k} \omega$ the maps (9.2) and (9.1) if $Z=\bar{S}$.

Definition 9-1. (cf. Def. 11.1 [4]) The $k^{\text {th }}$-logarithmic jacobian ideal of $(S, 0)$ is the $\mathcal{O}_{\bar{S}}$-module generated by the set $\left.\phi\left(\wedge^{k} \omega\left(\wedge^{k} \eta\left(\Omega_{S}^{k}\right)\right)\right) \wedge \bigwedge^{d-k} M\right) \subset \mathcal{O}_{\bar{S}}$. 
Proposition 9.2. (generalizing Theorem 3.3 of [17], cf. Prop. 11.2 [4]) For $k=$ $1, \ldots, d$ the $k^{\text {th }}$-logarithmic jacobian ideal of a q.o. hypersurface $(S, 0)$ is the monomial ideal $\mathcal{J}_{k}$ of $\mathbf{C}\left\{\sigma^{\vee} \cap M\right\}$ of Definition [5.2.

Proof. We denote by $\mathcal{J}_{k}^{\prime}$ the ideal generated by $\left.\phi\left(\wedge^{k} \omega\left(\wedge^{k} \eta\left(\Omega_{S}^{k}\right)\right)\right) \wedge \wedge^{d-k} M\right) \subset \mathcal{O}_{\bar{S}}$. For $I=\left(i_{1}, \ldots, i_{k}\right) \subset\{1, \ldots, d+1\}^{k}$ we analyze the images of the elements $d x_{I}:=$ $d x_{i_{1}} \wedge \ldots \wedge d x_{i_{k}}$, which generate $\Omega_{S}^{k}$ as a $\mathcal{O}_{S}$-algebra, by the homomorphism $\wedge^{k} \omega$. We have two possibilities.

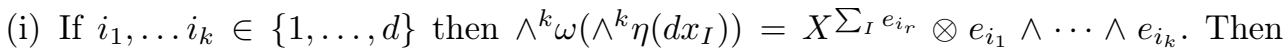
taking wedges with elements of $\wedge^{d-k} M$ and applying the homomorphism $\phi$ we obtain a generator of $\mathcal{J}_{k}^{\prime}$ equal to $X^{e_{i_{1}}+\cdots+e_{i_{k}}}$ if and only if $e_{i_{1}} \wedge \cdots \wedge e_{i_{k}} \neq 0$.

(ii) If one of the $i_{r}$, say $i_{k}$, is equal to $d+1$, then we set $n:=\min \{1 \leqslant j \leqslant g \mid$ $\left.e_{i_{1}} \wedge \cdots e_{i_{k-1}} \wedge \lambda_{j} \neq 0\right\} \cup\{\infty\}$. The image of $x_{d+1}$ in $\mathcal{O}_{\bar{S}}=\mathbf{C}\left\{\sigma^{\vee} \cap M\right\}$ is of the form $x_{d+1}=a+b$ where $a=\sum_{\lambda \geqslant \lambda_{n}} \beta_{\lambda} X^{\lambda}$ and $b=\sum_{\lambda \nsucceq \lambda_{n}} \beta_{\lambda} X^{\lambda}$. Then we have that $d x_{d+1}=d a+d b$ in $\Omega_{\bar{S}}$ hence $\wedge^{k} \omega\left(\wedge_{l=1, \ldots, k-1} d x_{i_{l}} \wedge d a\right)=0$ and

$$
\wedge^{k} \omega\left(\wedge^{k} \eta\left(d x_{I}\right)\right)=\wedge^{k} \omega\left(\wedge_{l=1, \ldots, k-1} d x_{i_{l}} \wedge d b\right) .
$$

Then taking wedges with elements of $\wedge^{d-k} M$ and applying the homomorphism $\phi$ we obtain a generator of $\mathcal{J}_{k}^{\prime}$ of the form:

$$
X^{e_{i_{1}}+\cdots+e_{i_{k-1}}+\lambda_{n}} \epsilon_{n}+\sum_{\text {finite }} X^{e_{i_{1}}+\cdots+e_{i_{k-1}}+v} \epsilon_{v}
$$

where $\epsilon_{\lambda_{n}}$ and $\epsilon_{v}$ are units in $\mathcal{O}_{\bar{S}}, v \in \sigma^{\vee} \cap M_{0}$ and $e_{i_{1}} \wedge \cdots \wedge e_{i_{k-1}} \wedge v \neq 0$.

The elements we obtain in (i) and (ii) are generators of $\mathcal{J}_{k}^{\prime}$. Since $M_{0}=\mathbf{Z} e_{1}+\cdots+\mathbf{Z} e_{d}$ the terms $X^{e_{i_{1}}+\cdots+e_{i_{k-1}}+v}$ belong to $\mathcal{J}_{k}^{\prime}$ hence we deduce that $\mathcal{J}_{k}=\mathcal{J}_{k}^{\prime}$.

\section{Examples}

Example 10·1. We compute the series $P_{\text {geom }}^{(S, 0)}(T)$ for a q.o. surface parametrized by a q.o. branch $\zeta$ with characteristic exponents $\lambda_{1}=(3 / 2,0), \lambda_{2}=(7 / 4,0)$ and $\lambda_{3}=$ $(2,1 / 2)$.

We have that $\sigma=\mathbf{R}_{\geqslant 0}^{2}$ and $\sigma^{\vee} \cap M \cong \mathbf{Z}_{\geqslant 0}^{2}$. It follows that $(S, 0)$ has smooth normalization thus $P_{\text {geom }}^{(\bar{S}, 0)}(T)=\left(1-\mathbf{L}^{2} T\right)^{-1}$. We denote by $\theta_{1}$ and $\theta_{2}$ the one-dimensional faces of $\sigma$. The plane curves $S_{\theta_{1}}$ and $S_{\theta_{2}}$ have multiplicities two and four respectively which determine the terms $P\left(S_{\theta_{1}}\right)$ and $P\left(S_{\theta_{2}}\right)$ by Remark 5.5. Notice that $\mathcal{D}_{1}=\emptyset$ thus

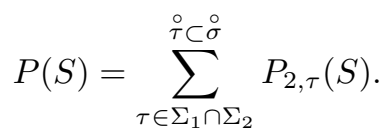

We determine this sum by computing the generating functions of the sets $\stackrel{\circ}{\tau} \cap N$ and applying the method of Proposition 9.5 and Section 12 of [4]. We get:

$$
\begin{gathered}
P_{2}(S)=\frac{(\mathbf{L}-1)^{2}}{1-\mathbf{L}^{2} T}\left(\frac{\mathbf{L}^{13} T^{17}}{(1-\mathbf{L} T)\left(1-\mathbf{L}^{12} T^{16}\right)}+\frac{\mathbf{L}^{2} T^{6}+\mathbf{L}^{4} T^{8}+\mathbf{L}^{6} T^{10}+\mathbf{L}^{8} T^{12}+\mathbf{L}^{10} T^{14}+\mathbf{L}^{12} T^{20}}{\left(1-\mathbf{L}^{12} T^{16}\right)\left(1-T^{4}\right)}\right. \\
\left.+\frac{\mathbf{L}^{2} T^{4}+\mathbf{L}^{4} T^{8}}{\left(1-T^{4}\right)\left(1-\mathbf{L}^{4} T^{4}\right)}+\frac{\mathbf{L}^{12} T^{16}}{1-\mathbf{L}^{12} T^{16}}+\frac{T^{4}}{1-T^{4}}\right) .
\end{gathered}
$$

We have that $P_{\text {geom }}^{(S, 0)}(T)=(1-T)^{-1}+P\left(S_{\theta_{1}}\right)+P\left(S_{\theta_{2}}\right)+P(S)$. The motivic volume is

$$
\mu\left(H_{S}\right)=\frac{1}{(1-\mathbf{L})\left(1-\mathbf{L}^{20}\right)}+\frac{-1}{1-\mathbf{L}^{20}}+\frac{1+\mathbf{L}^{6}+\mathbf{L}^{8}+\mathbf{L}^{10}+\mathbf{L}^{12}+\mathbf{L}^{14}+\mathbf{L}^{16}+\mathbf{L}^{18}}{\left(1-\mathbf{L}^{4}\right)\left(1-\mathbf{L}^{20}\right)} \text {. }
$$


Example 10.2. We describe the series $P_{\text {geom }}^{(Z(S), 0)}(T)$ associated with the monomial variety associated with the q.o. $\operatorname{surface}(S, 0)$ of example 10.1 (see Definition 3.5).

The semigroup $\Gamma$ is determined by the characteristic exponents. In this example the semigroup is generated by $e_{1}=(1,0), e_{2}=(0,1 / 2), e_{3}=(3 / 2,0), e_{4}=(13 / 4,0)$ and $e_{5}=(24 / 4,1 / 4)$. The lattice $M$ coincides with the one of $\bar{S}$. The dual cone of $\Gamma \mathbf{R}_{\geqslant 0}$ coincides with $\sigma$. Since the monomial curves $Z^{\Gamma \cap \theta_{1}^{\perp}}$ and $Z^{\Gamma \cap \theta_{2}^{\perp}}$ have multiplicities 2 and 4 respectively, we get $P\left(\Gamma \cap \theta_{i}^{\perp}\right)=P\left(S_{\theta_{i}}\right)$ for $i=1$, 2. Since $\mathcal{D}_{1}=\emptyset$ we obtain that $P(\Gamma)$ is obtained by a formula analogous to the right hand-side of (10.1), in terms of the subdivision associated to the logarithmic jacobian ideals of $Z^{\Gamma}$ (see 4 ). The subdivision obtained for the monomial variety is different that the one obtained for the q.o. singularity. We get:

$$
\begin{gathered}
P(\Gamma)=\frac{(\mathbf{L}-1)^{2}}{1-\mathbf{L}^{2} T}\left(\frac{\mathbf{L}^{51} T^{55}}{(1-\mathbf{L} T)\left(1-\mathbf{L}^{50} T^{54}\right)}+\frac{\mathbf{L}^{50} T^{54}}{1-\mathbf{L}^{50} T^{54}}+\frac{\mathbf{L}^{50} T^{58}+\sum_{k=3}^{26} \mathbf{L}^{2 k-4} T^{2 k}}{\left(1-\mathbf{L}^{50} T^{54}\right)\left(1-T^{4}\right)}+\right. \\
\left.\frac{T^{4}}{1-T^{4}}+\frac{\mathbf{L}^{2} T^{4}+\mathbf{L}^{4} T^{8}}{\left(1-T^{4}\right)\left(1-\mathbf{L}^{4} T^{4}\right)}\right) .
\end{gathered}
$$

Then we have that $P_{\text {geom }}^{(Z(S), 0)}(T)=(1-T)^{-1}+P\left(\Gamma \cap \theta_{1}^{\perp}\right)+P\left(\Gamma \cap \theta_{2}^{\perp}\right)+P(\Gamma)$, and it is easy to see that $P_{\text {geom }}^{(S, 0)}(T) \neq P_{\text {geom }}^{\left(Z^{\Gamma}, 0\right)}(T)$.

See 3 for explicit examples of geometric motivic Poincaré series of toric or q.o. singularities of dimensions two or three.

\section{REFERENCES}

[1] Abhyankar, S.S., On the ramification of algebraic functions. Amer. J. Math., 77. (1955), 575-592.

[2] Artal Bartolo, E., Cassou-Noguès, Pi., Luengo, I. and Melle Hernández, A., Quasi-ordinary power series and their zeta functions. Mem. Amer. Math. Soc. 178 (2005), no. 841, 1-85.

[3] Cobo Pablos, H. Arcs and motivic Poincaré series, Tesis Doctoral, Universidad Complutense de Madrid, 2009.

[4] Cobo Pablos, H. and González Pérez, P.D. Motivic Poincaré series, toric singularities and logarithmic jacobian ideals. Preprint 2009.

[5] Denef, J. And Loeser. F. Germs of arcs on singular algebraic varieties and motivic integration. Invent. Math. 135, 1, (1999), 201-232.

[6] Denef, J. And Loeser. F. Geometry on arc spaces of algebraic varieties. European Congress of Mathematics, Vol. I (Barcelona, 2000), 327-348, Progr. Math., 201, Birkhäuser, Basel, 2001

[7] Denef, J. and Loeser. F. Definable sets, motives and p-adic integrals J. Amer. Math. Soc. 14, 4, (2001) 429-469.

[8] Denef, J. And Loeser. F., Motivic integration, quotient singularities and the McKay correspondance, Compositio Math. 131 (2002), 267-290.

[9] Denef, J. And Loeser. F., On some rational generating series occuring in arithmetic geometry, in Geometric Aspects of Dwork Theory, edited by A. Adolphson, F. Baldassarri, P. Berthelot, N. Katz and F. Loeser, volume 1, de Gruyter, 509-526 (2004).

[10] Ein, L. and Mustaţa, M. Jet Schemes and Singularities, Algebraic geometry-Seattle 2005, 505-546, Proc. Sympos. Pure Math., 80, Part 2, Amer. Math. Soc., Providence, RI, 2009.

[11] Ewald, G. Combinatorial Convexity and Algebraic Geometry, Springer-Verlag, 1996.

[12] Fulton, W. Introduction to Toric Varieties, Annals of Math. Studies (131), Princenton University Press, 1993.

[13] Gau, Y-N., Embedded Topological classification of quasi-ordinary singularities, Memoirs of the American Mathematical Society 388, 1988.

[14] Goldin, R; Teissier, B. Resolving singularities of plane analytic branches with one toric morphism. Resolution of singularities (Obergurgl, 1997), 315-340, Progr. Math., 181, Birkhäuser, Basel, 2000

[15] González Pérez P.D., The semigroup of a quasi-ordinary hypersurface, J. Inst. Math. Jussieu, (2003), 2 (3), 383-399. 
[16] González Pérez, P.D., Toric embedded resolutions of quasi-ordinary hypersurface singularities, Ann. Inst. Fourier (Grenoble), 53 (6), (2003), 1819-1881.

[17] González PÉrez, P.D. Logarithmic jacobian ideals, quasi-ordinary hypersufaces and equisingularity, preprint 2009.

[18] González Pérez, P.D.; Gonzalez-Sprinberg, G. Analytical invariants of quasiordinary hypersurface singularities associated to divisorial valuations. Kodai Math. J. 27 (2004), no 2, 164-173.

[19] González Pérez, P.D., McEwan, L. J., And Némethi, A.; The zeta-function of a quasi-ordinary singularity. II; Topics in algebraic and noncommutative geometry (Luminy/Annapolis, MD, 2001), 109-122, Contemp. Math., 324, Amer. Math. Soc., Providence, RI, 2003.

[20] González Pérez, P.D. and Hernando, F. Quasi-ordinary singularities, essential divisors and Poincaré series, J. London Math. Soc., (2009), doi: 10.1112/jlms/jdp014.

[21] Greuel, G.-M. ; Lossen, C. And Shustin, E., Introduction to singularities and deformations. Springer Monographs in Mathematics. Springer, Berlin, 2007.

[22] Greenberg, M.J. Rational points in Henselian discrete valuation rings. Inst. Hautes Études Sci. Publ. Math. No. 31, 1966, 59-64.

[23] Ishit, S. The arc space of a toric variety, J. Algebra, Volume 278 (2004), 666-683.

[24] Ishil, S. Arcs, valuations and the Nash map. J. Reine Angew. Math. 588 (2005), 71-92.

[25] IsHit, S., The local Nash problem on arc families of singularities, Ann. Inst. Fourier (Grenoble). 56 (2006), no. 4, 1207-1224.

[26] Ishit, S, Jet schemes, arc spaces and the Nash problem. C. R. Math. Acad. Sci. Soc. R. Can. 29 (2007), no. 1, 1-21

[27] Jung, H.W.E., Darstellung der Funktionen eines algebraischen Körpers zweier unabhaängigen Veränderlichen $x, y$ in der Umgebung einer stelle $x=a, y=b$, J.Reine Angew. Math., 133 (1908), 289-314.

[28] Lejeune-Jalabert, M. and Reguera, A. The Denef-Loeser series for toric surface singularities. Proceedings of the International Conference on Algebraic Geometry and Singularities (Spanish) (Sevilla, 2001). Rev. Mat. Iberoamericana 19 (2003), no 2, 581-612.

[29] Lipman, J., Topological invariants of quasi-ordinary singularities, Memoirs of the American Mathematical Society 388, 1988.

[30] Lipman, J., Resolution of singularities (Obergurgl, 1997), 485-505, Progr. Math., 181, Birkhäuser, Basel, 2000.

[31] Looijenga, E., Motivic measures, Séminaire Bourbaki, Exposé 874, Astérisque 276, (2002), 267-297.

[32] McEwan, L.J. And NÉmethi, A.; Some conjectures about quasi-ordinary singularities; Topics in algebraic and noncommutative geometry (Luminy/Annapolis, MD, 2001), 185193, Contemp. Math., 324, Amer. Math.Soc., Providence, RI, 2003.

[33] McEwan, L.J. And NÉmethi, A. ; The zeta function of a quasi-ordinary singularity; Compos. Math. 140 (2004), no. 3, 667-682.

[34] Nicaise, J. Motivic generating series for toric surface singularities Math. Proc. Camb. Phil. Soc. 138 (2005), 383-400.

[35] Nicaise, J. Arcs and resolution of singularities Manuscripta Math. 116 (2005), 297-322.

[36] OdA, T. Convex Bodies and Algebraic Geometry, Annals of Math. Studies (131), SpringerVerlag, 1988.

[37] Popescu-Pampu, P. On the analytical invariance of the semigroups of a quasi-ordinary hypersurface singularity. Duke Math. J. 124 (2004), no. 1, 67-104.

[38] Popescu-Pampu, P. On higher dimensional Hirzebruch-Jung singularities. Rev. Mat. Complut. 18 (2005), no. 1, 209-232.

[39] Teissier, B., Valuations, deformations, and toric geometry. Valuation theory and its applications, Vol. II (Saskatoon, SK, 1999), 361-459, Fields Inst. Commun., 33, Amer. Math. Soc., Providence, RI, 2003.

[40] G. Rond Séries de Poincaré motiviques d'un germe d'hypersurface irréductible quasiordinaire, Astérisque. 157 (2008), 371-396.

[41] Veys, W. Arc spaces, motivic integration and stringy invariants, Advanced Studies in Pure Mathematics 43, Proceedings of "Singularity Theory and its applications, Sapporo (Japan), 16-25 september 2003" (2006), 529-572. 